\title{
Downregulation of the $P H L D A 1$ gene in IMR-32 neuroblastoma cells increases levels of Aurora A, TRKB and affects proteins involved in apoptosis and autophagy pathways
}

\author{
MAŁGORZATA DURBAS, IRENA HORWACIK, ELŻBIETA BORATYN and HANNA ROKITA \\ Laboratory of Molecular Genetics and Virology, Faculty of Biochemistry, \\ Biophysics and Biotechnology, Jagiellonian University, 30-387 Kraków, Poland
}

Received February 25, 2016; Accepted May 20, 2016

DOI: $10.3892 /$ ijo.2016.3572

\begin{abstract}
We have recently shown that mRNA and protein of PHLDAl (pleckstrin-homology-like domain family A, member 1) were by far the most upregulated molecules upon treatment of IMR-32 cells with the anti-GD2 ganglioside monoclonal antibody 14G2a. Hence, we decided to study functions of PHLDA1 using human neuroblastoma IMR-32 cells as a model. Here, we show that constitutive expression of mRNA and protein of the PHLDAl gene in IMR-32 cells was inversely correlated with transcript of the AURKA gene and Aurora A oncoprotein. Next, we silenced PHLDA1 expression in IMR-32 cells using an shRNA interference method. We report that IMR-32 cells with stable downregulation of PHLDA1 showed enhanced cellular ATP levels and an increase in mitochondrial membrane potential, as compared to control and non-transduced cells. We demonstrated that downregulation of PHLDAl leads to a significant increase in expression of Aurora A and TRKB that are markers of poor prognosis in neuroblastoma. Also, we measured an increase in Aurora A and Akt kinases phosphorylation in the cells. Most importantly, PHLDA1-silenced cells were less susceptible to apoptosis than control cells, as shown by the lower expression of cleaved caspase- 3 and PARP as well as a decreased activity of caspase-3 and -7. Our study negatively correlates expression of PHLDA1 and Aurora A in IMR-32 cells and sheds new light on functions of PHLDA1 in the neuroblastoma tumor cells,
\end{abstract}

Correspondence to: Dr Irena Horwacik, Laboratory of Molecular Genetics and Virology, Faculty of Biochemistry, Biophysics and Biotechnology, Jagiellonian University, Gronostajowa 7, 30-387 Kraków, Poland

E-mail: irena.horwacik@uj.edu.pl

Abbreviations: Ab, antibody; FBS, fetal bovine serum; GD2, GD2 ganglioside; mAb, monoclonal antibody; MMP, mitochondrial membrane potential; RT-qPCR, quantitative RT-PCR

Key words: neuroblastoma, PHLDA1, Aurora A, apoptosis, ganglioside GD2, monoclonal antibody suggesting its role as a pro-apoptotic protein. Additionally, our results show possible links of the protein to regulation of features of mitochondria and formation of autophagosomes.

\section{Introduction}

PHLDA1 was identified as a gene involved in Fas (CD95) expression and apoptosis induced after an anti-TCR antibody binding to mouse $\mathrm{T}$ cell hybridoma cells (1). In humans the gene is located on the long arm of the chromosome 12 [12q15 (2)] and encodes a 401 amino acid (aa) protein (45 kDa) named pleckstrin-homology-like domain family A, member 1 (PHLDA1, synonyms include: DT1P1B11, PHRIP, 'prolinehistidine rich protein', TDAG51, based on www.genecards. org).

Several research groups have aimed to characterize involvement of PHLDA1 in biology of cancer cells, including its function in apoptosis. Thus, Neef et al measured levels of mRNA of PHLDAl in three sets of cell lines, derived from paired samples of primary and metastatic melanoma tumors, and reported that $P H L D A 1$ was downregulated in the latter. PHLDA1 was detected by the authors in benign melanocytic nevi, and its level was shown to decrease with progression of malignant melanomas. Also, constitutive expression of the PHLDA1 protein in a melanoma cell line Mel Rif has led to cell growth reduction along with an increase in basal apoptosis and sensitivity to doxorubicin and camptothecin (3). In 2006, Hayashida et al showed that PHLDAl is a heat shock inducible gene regulated by HSF1 and when overexpressed in HeLa cells has pro-apoptotic functions (4). In yet another report, Nagai et al have reported that downregulation of PHLDA1 is a strong predictor of poor outcome in patients with breast cancer (5). On the contrary, Coutinho-Camillo et al reported that PHLDA1 and PAR-4 were associated with an advanced clinical stage in oral squamous cell carcinomas (6). In some in vitro models, i.e., apoptosis induced by oxidative stress in mouse embryonic fibroblasts and apoptosis induced with hydrogen sulfide in an oral cancer cell line Ca9-22, the lack of PHLDA1 correlated with activation of caspase-3, suggesting that the protein is a suppressor of apoptosis $(7,8)$. Hence, it can be concluded that functions of PHLDA1 in regulation of cancer cell fate are not yet definitely determined and should be further investigated. 
Examples of proteins interacting with PHLDA1 have already been identified. Thus, HSP110, HSP70, HSP40 were shown to directly bind to PHL domain of PHLDA1 (4). In a yeast two hybrid system three proteins involved in regulation of translation, including RPL14, were found to bind PHLDA1 (9). In 2011, Johnson et al have shown that the mitotic kinase Aurora A, an oncoprotein overexpressed in the majority of breast cancer tumors, and PHLDA1 associate regulating each other in MDA-MB-231 breast cancer cells (10). In neuroblastoma, the most common extra-cranial tumor of childhood, Aurora A (encoded by AURKA) and MYCN are partners in driving the malignancy and negative prognostic factors $(11,12)$. Importantly, this interaction provides an opportunity to target high risk $M Y C N$-amplified neuroblastoma tumors with Aurora A inhibitors $(13,14)$, as MYCN due to its function of a transcription factor is viewed as 'undruggable' (15).

GD2 ganglioside is a marker of neuroblastoma, involved in maintaining malignant phenotype of the tumor. Also, the glycolipid is the target for Dinutuximab, a chimeric human/mouse antibody (Unituxin ${ }^{\mathrm{TM}}$, ch14.18), approved in 2015 by FDA to treat patients with high risk neuroblastoma (16). Previously, we have reported that treatment of $M Y C N$-amplified IMR-32 neuroblastoma cells with the anti-GD2 ganglioside mouse monoclonal antibody $14 \mathrm{G} 2 \mathrm{a}(\mathrm{mAb})$, sharing a paratope with ch14.18, and a specific Aurora A inhibitor MK-5108 increased expression of PHLDA1, when used alone or combined together (17). Incubation of the mAb with IMR-32 neuroblastoma cell line led to cell death with involvement of apoptosis $(17,18)$ and this correlated with a decrease in the Aurora A protein level and its activating phosphorylation, a decrease of the cytoplasmic level of MYCN, and an increase in the p53 level (17).

Based on our prior findings, we aimed to further study roles of PHLDA1 in our model. Thus, we set goals to silence PHLDAl expression in the cell line and investigated effects of that on Aurora A and its direct or indirect targets (19), e.g., Akt, MYCN, p53, PARP, p21, p27 (among others). Pathways of molecular cross talk between apoptosis, programmed necrosis, and mitochondrial autophagy (mitophagy) signaling have recently been advanced by Dorn and Kitsis. They postulate that these processes are "components of a unified and integrated quality control mechanism' (20). Encouraged by the aforementioned accounts, we sought to determine how PHLDA1 is interlinked to both apoptosis and autophagy pathways in our model.

Here, we report that IMR-32 cells with stable downregulation of PHLDA1 showed enhanced cellular levels of ATP, increased mitochondrial membrane potential (MMP), and were less susceptible to apoptosis, as compared to control cells. Also, we demonstrated that downregulation of PHLDA1 leads to a significant increase in the expression of AURKA. Furthermore, we measured increased phosphorylation of p-Aurora A (Thr288) and p-Akt (Thr308), and lower levels of cleaved PARP and caspase-3 in the cells. Additionally, we correlated expression of products of two genes regulating cell cycle, i.e., $C D K N 1 A$ and $C D K N 1 B$ and showed that mRNA of the $C D K N 1 A$ gene and the p27 protein are reduced in the PHLDA1-silenced settings. Next, profiles of transcripts of some differentiation markers, related to prognosis of neuroblastoma outcome such as NTRK1 (encoding TRKA) and NTRK2 (encoding TRKB) were altered in our model. Also the protein levels of TRKB were increased in the clones with PHLDA1 downregulation. Our data show an inverse correlation of expression of PHLDA1 and Aurora A in some cell lines and shed new light on functions of PHLDA1 in neuroblastoma tumor cells, suggesting a role as a pro-apoptotic protein. Also, our results show possible links of the protein to regulation of features of mitochondria and formation of autophagosomes.

\section{Materials and methods}

Cell culture and antibody purification. The following cell lines were used: IMR-32 (ATTC, Lomianki, Poland, CCL-127), LA-N-1 (ECACC, Sigma-Aldrich, Poznan, Poland, 06041201), CHP-134 (ECACC, 06122002), LA-N-5 (ACC 673, LeibnizInstitut DSMZ - Deutsche Sammlung von Mikroorganismen und Zellkulturen GmbH, Braunschweig, Germany), Kelly (ACC 355, DSMZ), SK-N-SH (ATTC, HTB-11), HTLA230 (kindly provided by Dr Lizzia Raffaghello, Laboratory of Oncology, G. Gaslini Institute, Genova, Italy). The hybridoma cell line producing the 14G2a mAb (IgG2a), binding to GD2, was kindly provided by Dr R.A. Reisfeld (Scripps Institute, La Jolla, CA, USA). Cells were cultured as described in detail (17). The 14G2a mAb was purified from FBS (fetal bovine serum) free cell culture supernatant as described in detail (17).

Lentiviral transduction. IMR-32 $\left(2.5 \times 10^{5}\right)$ cells were plated in $2 \mathrm{ml}$ of complete medium in a 12 -well plate, $24 \mathrm{~h}$ prior to viral infection. On the day of infection media were replaced with $2 \mathrm{ml}$ of complete medium with Polybrene ${ }^{\circledR}$ (sc-134220, Santa Cruz Biotechnology, Dallas, TX, USA) at the final concentration of $5 \mu \mathrm{g} / \mathrm{ml}$. Next, cells were infected by adding $40 \mu \mathrm{l}$ of the shRNA Lentiviral Particles stock (sc-36631-V, Santa Cruz Biotechnology) to the culture (for the PHLDAl gene silencing) and $45 \mu \mathrm{l}$ of the shRNA Lentiviral Particles stock (sc-29731-V, Santa Cruz Biotechnology) to the culture (for the AURKA gene silencing). Additionally, for both PHLDAl and AURKA shRNA silencing one well with cells was transduced with Control shRNA Lentiviral Particles (sc-108080, Santa Cruz Biotechnology) and one well was used as non-transduced control cells. Finally, separate wells with cells were also transduced with copGFP Control Lentiviral Particles (sc-108084, Santa Cruz Biotechnology) for measuring transduction efficiency. On the third day media were replaced with $2 \mathrm{ml}$ of complete medium (without Polybrene). On the fourth day cells were split and to both PHLDA1- and AURKA-silenced and mock cells, puromycin dihydrochloride (sc-108071, Santa Cruz Biotechnology) was added to the fresh medium to the concentration of $5 \mu \mathrm{g} / \mathrm{ml}$. Medium was replaced with fresh puromycin-containing medium every 3-4 days, until resistant colonies were identified. Then these colonies were picked with small pipette tips, expanded and assayed for effects of stable shRNA expression by RT-qPCR and western blot analyses to derive PHLDA1-stably silenced clones. To derive AURKAtransiently silenced cell pools, cells were collected 4 days after adding puromycin dihydrochloride.

Flow cytometry analyses. The 14G2a mAb (IgG2a) was used to detect presence of GD2 ganglioside on cell clones (WT, Mock1, S2, S4). The PK136 mAb (IgG2a) was used as an isotype control (17). Binding of the antibodies were 
detected using mouse Ig-specific FITC-conjugated goat $\mathrm{F}(\mathrm{ab})_{2}$ fragments (cat. no. 55526, Cappel, MP Biomedicals, LLC, Warszawa, Poland), a detailed protocol can be seen in ref. 17. Cell cycle analyses were performed using the protocols for adherent cells described in detail (21). For mitochondrial potential analyses MitoTracker ${ }^{\circledR}$ Red CMXRos was used (cat. no. M7512, Invitrogen, Warszawa, Poland) and the staining protocol was optimized according to the manufacturer's instructions. Briefly, cells $\left(0.5 \times 10^{6}\right.$ cells per tube) were incubated for $25 \mathrm{~min}$ at $37^{\circ} \mathrm{C}$ with $500 \mathrm{nM}$ of the dye in complete medium. Then, cells were washed once with medium with $2 \%$ FBS and collected by centrifugation. Cells were resuspended in medium with $2 \%$ FBS prior to analysis. To determine pools containing mostly intact cells that were used for analyses of mitochondrial membrane potential, first separate samples of cells stained with propidium iodide were analyzed (17). Samples were run in duplicates. In all experiments samples were analyzed using BD $^{\text {TM }}$ LSR II with BD FACSDiva software (BD Biosciences, Warszawa, Poland).

Measurements of ATP levels in cell cultures. Respective IMR-32 clones $\left(5 \times 10^{3}\right)$ (WT, Mock1, S1, S2, S4) were cultured for 6 days in $100 \mu \mathrm{l}$ of complete medium in a 96-well plate. Comparison of metabolic activity was made between PHLDA1-silenced clones, mock and wild-type cells at selected time-points (1-6 days) by measuring cellular ATP contents using ATPlite - luminescence ATP detection assay system (cat. no. 6016947, Perkin-Elmer, Warszawa, Poland) according to the manufacturer's protocol. Studies were run in triplicates. Relative ATP levels were calculated as follows: relative ATP level = ATP level at specific time-point / ATP level at day 1. Samples were analyzed using the Infinite M200 reader (TECAN, Männedorf, Switzerland).

Measurements of levels of $\mathrm{LDH}$ and activity of caspases-3 and -7 in cell cultures. Respective IMR-32 clones $\left(5 \times 10^{3}\right)$ (WT, Mock1, S2, S4) were cultured for 6 days in $150 \mu 1$ of complete medium in a 96-well plate (samples were analyzed in triplicates). On days 1 and $6,100 \mu 1$ of culture supernatant was transferred to a separate plate and LDH measurements were performed using Cytotoxicity Detection kit (cat. no. 11644793001, Roche, Warszawa, Poland) according to the manufacturer's protocol. Signals were analyzed using Versa max microplate reader (Molecular Devices, Sunnyvale, CA, USA). For measurements of activity of caspases-3 and -7 remaining cell cultures were analyzed using Caspase-Glo ${ }^{\circledR}$ 3/7 assay (cat. no. G8091, Promega, Warszawa, Poland) according to the manufacturer's protocol. All samples were analyzed in triplicates. Signals were analyzed using the Infinite M200 reader.

Antibody and drug treatments. WT, Mock1, S1, S2, S4 clones were incubated with the $14 \mathrm{G} 2 \mathrm{a} \mathrm{mAb}(40 \mu \mathrm{g} / \mathrm{ml})$ or PBS (control cells) for $1 \mathrm{~h}$ on ice. Next, the cells were seeded on 96 -well plates ( $2 \times 10^{4}$ cells $/ 100 \mu \mathrm{l} /$ well), and incubated for 1-6 days, and then the ATP levels were determined. To isolate proteins (whole cell extracts), cells were cultured in 6 -well plates $\left(1 \times 10^{6}\right.$ cells in $5 \mathrm{ml}$ of culture medium for all the clones) and treated for $48 \mathrm{~h}$ with the $14 \mathrm{G} 2 \mathrm{a}$ mAb or PBS. In some experiments Aurora A kinase inhibitor (MK-5108, cat. no. S2770, Selleck,
STI, Poznan, Poland) was used in range of concentrations from 0.1 to $1 \mu \mathrm{M}$. Two dual PI3K/mTOR dual inhibitors were also used. BEZ-235 (NVP-BEZ235, cat. no. S1009, Selleck) was used in the range from 0.01 to $1 \mu \mathrm{M}$ and LY294002 (\#9901, Cell Signaling, Lab-JOT, Warszawa, Poland) was used in concentrations from 0.1 to $30 \mu \mathrm{M}$. Inhibitors were added to cell cultures for $72 \mathrm{~h}$, and then ATP levels were determined. Control cells were treated with equivalent volume of DMSO or water (solvents for the inhibitors).

RNA isolation and quantitative RT-PCR (RT-qPCR). Samples of total RNA were isolated from cell clone using TRI-Reagent ${ }^{\circledR}$ (cat. no. TR118, Lab Empire, Rzeszow, Poland) as described in the manufacturer's protocol (Molecular Research Center, Inc., Cincinnati, OH, USA) as can be seen in more detail in ref. 17. Total RNA ( $2 \mu \mathrm{g})$ was used for reaction with reverse transcriptase (M-MLV reverse transcriptase, cat. no. M1302, Sigma-Aldrich), using Oligo(dT) 20 Primer (cat. no. 1841802, Invitrogen). The Eco (Illumina) system and KAPA SYBR FAST qPCR Master Mix (Kapa Biosystems, cat. no. KK4602, Polgen, Lodz, Poland) were used for RT-qPCR (cDNA dilutions were optimized within the range of 20-100 times). For normalization of samples, the amount of cDNA of eukaryotic translation elongation factor 2 (eEF-2) or ribosomal protein S13 (RPS13) were measured. Unless otherwise stated, primers were designed using Primer-BLAST (http://www.ncbi.nlm. nih.gov/tools/primer-blast/). Following primers were used: PHLDAl (F: TGCCTGAAAGGGGCAGCTCC, R: TGATCT GGTGCGGGGCGGA) (17); AURKA (F: GTCAAGTCCCCT GTCGGTTC, R: CGGTCCATGATGCCTCTAGC); $M Y C N$ (F: ACCACAAGGCCCTCAGTACCT, R: GTGGTGACAGC CTTGGTGTTG) (22); CDKNIA (F: CGATGGAACTTCGAC TTTGTCA, R: GCACAAGGGTACAAGACAGTG, previously described (23); CDKN1B (F: GAAGCGACCTGCAACCGA CGATT, R: CAGGCTTCTTGGGCGTCTGCTC) (24); IDI (F: TCAGCACCCTCAACGGCGAGAT, R: CCAGGTACCC GCAAGGATGGGA) (25); ID2 (F: TCAGCCTGCATCAC CAGAGA, R: CTGCAAGGACAGGATGCTGAT); NTRK1 [F: GGGCCTCTCCTTACAGGAAC, R: CTGACTGCTCCA GCTCTGTG, previously described (26)]; NGF [F: TCCGG ACCCAATAACAGTTT, R: CAGTGTCAAGGGAATGC TGA, previously described (26)]; NTRK2 [F: GGGACACCAC GAACAGAAGTA, R: ACCACAGCATAGACCGAGAGA, previously described(27)]; $B D N F$ [F: TAACGGCGGCAGACA AAAAGA, R: GAAGTATTGCTTCAGTTGGCCT, previously described (28)]; DKK3 [F: ACAGCCACAGCCTGGTGTA, R: CCTCCATGAAGCTGCCAAC, previously described (29)]; $e E F-2$ (F: GACATCACCAAGGGTGTGCAG, R: TCAGCA CACTGGCATAGAGGC) (30); RPS13 (F: TCGGCTTTACC CTATCGACGCAG, R: ACGTACTTGTGCAACACCATG TGA) (31). Sample quantification was performed in triplicates and results were analyzed using the ' $\Delta \Delta \mathrm{Cq}$ ' relative quantification method.

Protein extract isolation and immunoblotting. Whole cell extracts were obtained according to the TRI-Reagent method or using RIPA buffer (25 mM Tris- $\mathrm{HCl}$ pH 7.6, $150 \mathrm{mM} \mathrm{NaCl}$, $1 \%$ sodium deoxycholate, $0.1 \%$ SDS) supplemented with Halt Phosphatase Cocktail (cat. no. 1862495, Thermo Scientific, Warszawa, Poland) and cOmplete (cat. no. 11697498001, 

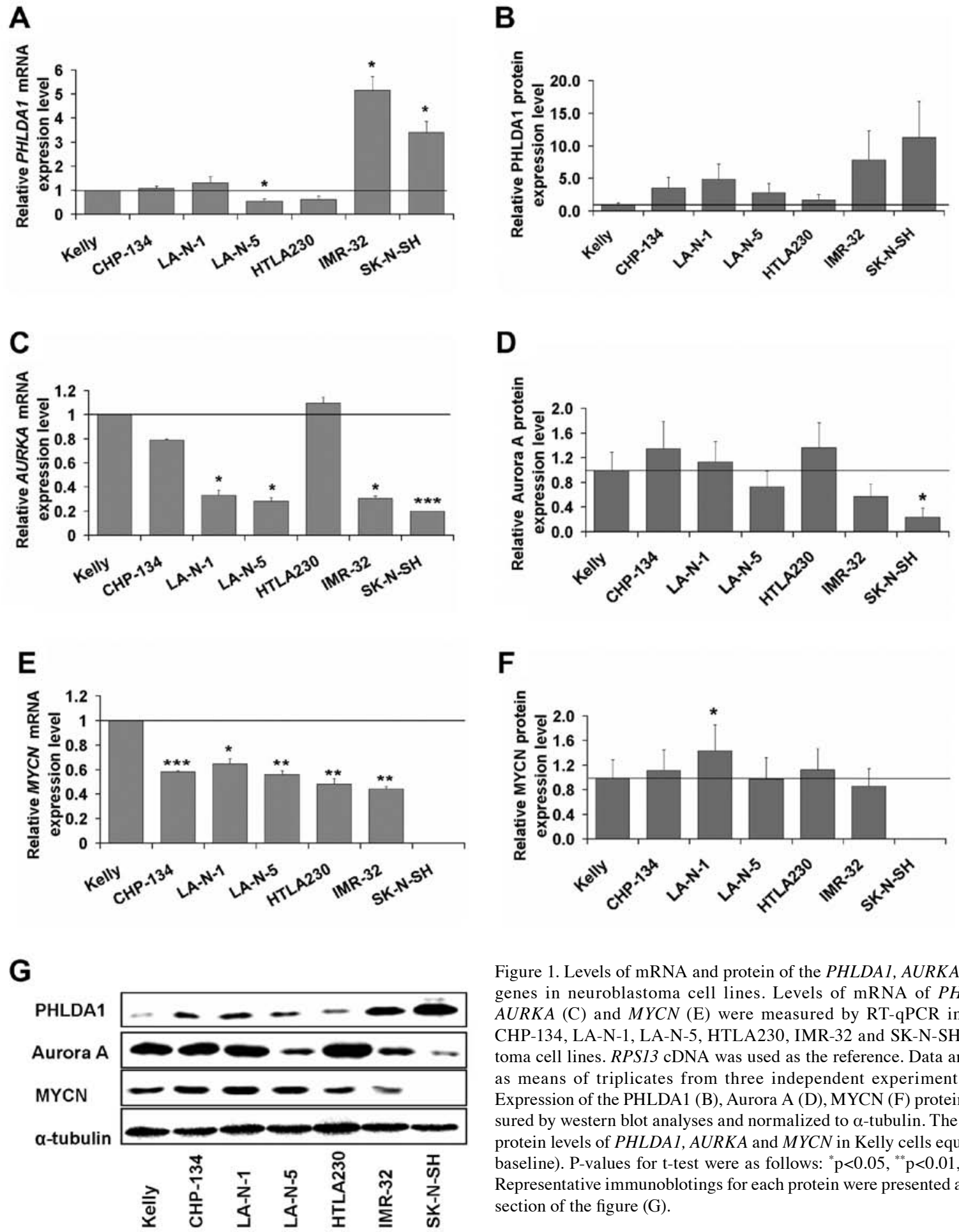

Figure 1. Levels of mRNA and protein of the PHLDA1, AURKA and MYCN genes in neuroblastoma cell lines. Levels of mRNA of PHLDA1 (A), AURKA (C) and MYCN (E) were measured by RT-qPCR in the Kelly, CHP-134, LA-N-1, LA-N-5, HTLA230, IMR-32 and SK-N-SH neuroblastoma cell lines. RPS13 cDNA was used as the reference. Data are presented as means of triplicates from three independent experiments $( \pm$ SEM). Expression of the PHLDA1 (B), Aurora A (D), MYCN (F) proteins was measured by western blot analyses and normalized to $\alpha$-tubulin. The mRNA and protein levels of PHLDA1, AURKA and MYCN in Kelly cells equals 1 (black baseline). P-values for t-test were as follows: ${ }^{*} \mathrm{p}<0.05,{ }^{* *} \mathrm{p}<0.01,{ }^{* * *} \mathrm{p}<0.001$ Representative immunoblotings for each protein were presented as a separate section of the figure $(\mathrm{G})$.

Roche), and the protein content in cell extracts was measured using BCA method (32). Western blot analyses were performed as previously described in detail (17). The first group of antibodies $(\mathrm{Ab})$ was from Cell Signaling Technology. Dilutions of all rabbit monoclonal anti-human antibodies were 1:1,000 for: anti-Aurora A Ab (\#4718), anti-phospho-Aurora A (Thr232)/ Aurora B (Thr232)/Aurora C (Thr198) Ab (\#2914), anti-phosphoAkt (Thr308) Ab (\#2965); anti-phospho-mTOR (Ser2448) Ab (\#5536), anti-cleaved caspase-3 Ab (\#9664), anti-cleaved PARP $\mathrm{Ab}$ (\#5625), anti-LC3A/B Ab (\#12741), anti-Atg7 Ab (\#8558), anti-HSF-1 Ab (\#4356), anti-HSP40 Ab (\#4871), anti- $\alpha$-tubulin

$\mathrm{Ab}$ (\#2125), anti-p21 Ab (\#2947), anti-p27 Ab (\#2552), anti-p53 (\#2527), anti-TRKB Ab (\#4603). The second group of antibodies was from Santa Cruz Biotechnology (USA) and their dilutions were as follows: mouse monoclonal anti-PHLDA1 $\mathrm{Ab}, \mathrm{sc}-23866,(1: 500)$; rabbit polyclonal anti-MYCN Ab, sc-791 (1:1,000). Mouse anti-human GAPDH antibody, G8795, was used in dilution 1:40,000 (Sigma-Aldrich). Following HRP-conjugated antibodies were applied: goat anti-rabbit IgG antibodies (Cell Signaling Technology), \#7074 (1:2,000) or rabbit anti-mouse IgG antibodies (Sigma-Aldrich), A-9044 $(1: 40,000)$. Immunoreactive bands were visualized by the ECM 
reagent (enhanced chemiluminescence method, Immobilon Western HRP Substrate, cat. no. WBKLS05000, Millipore, Warszawa, Poland) according to the manufacturer's protocol. Intensity of bands was quantified (Quest Spot Cutter, Quantity One Analysis Software, Bio-Rad) after detection of chemiluminescence with the MicroChemi system (DNR Bio-Imagining Systems Ltd., Jerusalem, Israel). Glyceraldehyde 3-phosphate dehydrogenase (GAPDH) or $\alpha$-tubulin were used as reference proteins for normalization of signals of proteins of interest in samples. In figures, levels of the protein expression in control samples were set as 1 .

Statistical analyses. Data shown on graphs are presented as means \pm SEM (a standard error of the mean). All experiments were repeated at least three times. Series of pairwise tests (t-test), comparing, e.g., WT clones with other clones, or means of control cells with treated cells were applied to measure statistical significance [p-values: $\mathrm{p}<0.05\left(^{*}\right), \mathrm{p}<0.01\left(^{* *}\right)$, $\left.\mathrm{p}<0.001\left(^{* * *}\right)\right]$. Statistical analyses were performed with the Excel software (Microsoft, USA).

\section{Results}

Characterization of levels of PHLDAl and Aurora A in human neuroblastoma cell lines. We analyzed expression levels of PHLDA1, MYCN, Aurora A in several MYCN-amplified neuroblastoma cell lines i.e., Kelly, CHP-134, LA-N-1, LA-N-5, HTLA230, IMR-32 and in a MYCN-non amplified SK-N-SH neuroblastoma cell line. For the PHLDAl gene, we observed by far the highest mRNA (Fig. 1A) and protein (Fig. 1B and $\mathrm{G}$ ) expression levels in IMR-32 and SK-N-SH cells. Thus, for IMR-32 and SK-N-SH cells the mean mRNA levels were $5.2( \pm 0.6)$ and $3.4( \pm 0.5)$ times statistically significantly higher than in Kelly cells (set as control, equal to 1), respectively. For the protein levels, in IMR-32 and SK-N-SH cells, the means were $7.9( \pm 4.7)$ and $11.3( \pm 5.5)$ times higher than in Kelly, respectively. In other cell lines, i.e., CHP-134, LA-N-1, LA-N-5, HTLA230, the mean expression levels ranged from 0.5 to 1.3 for mRNA of the PHLDAl gene and from 1.0 to 4.9 for the PHLDA1 protein, as compared to Kelly cells.

Further, we examined possible correlations of expression profiles of PHLDA1, AURKA and MYCN (mean values are shown on Fig. 1). The highest levels of mRNA of AURKA (Fig. 1C) were observed in Kelly, HTLA230 and CHP-134 cells and accompanied by low levels of mRNA of PHLDA1. The opposite results were measured for IMR-32 and SK-N-SH cells (Fig. 1A and C). For cell lines such as LA-N-1, LA-N-5, IMR-32 and SK-N-SH, expression of AURKA transcript was significantly lower i.e., $0.33( \pm 0.11), 0.28( \pm 0.03), 0.31( \pm 0.11)$, $0.20( \pm 0.02)$, respectively (as compared to signal from the Kelly cells, set as 1). Aurora A protein level (Fig. 1D and G) was the highest in CHP-134 (1.35 \pm 0.25$)$ and HTLA230 (1.36 \pm 0.10$)$ cells. Among all cell lines analyzed, the highest mRNA level of MYCN (Fig. 1E) was observed in Kelly cells (set as control, equal to 1), while in other cell lines the expression was significantly lower (in the range from $0.44 \pm 0.02$ to $0.65 \pm 0.04)$. However, levels of MYCN protein expression (Fig. 1F and G) were comparable for majority of neuroblastoma lines tested (ranged from $0.86 \pm 0.16$ to $1.13 \pm 0.13$ ), with exception of LA-N-1, for which significantly increased expres-
Table I. Flow cytometry analysis of DNA content in IMR-32 clones with downregulation of PHLDA1 (S2, S4) and WT and Mock1 clones. ${ }^{\mathrm{a}}$

\begin{tabular}{lrrrr}
\hline & WT & Mock1 & S2 & S4 \\
\hline Apoptotic & 4.6 & 4.5 & 2.5 & 2.3 \\
G0/G1 & 64.9 & 64.8 & 70.0 & 69.6 \\
S & 17.7 & 18.0 & 16.2 & 16.3 \\
G2/M & 13.5 & 13.5 & 12.3 & 12.8 \\
\hline
\end{tabular}

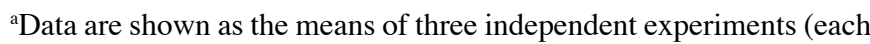
run in duplicates).

sion $(1.44 \pm 0.08)$ was noted. As reported in the literature (33) for $M Y C N$-non amplified SK-N-SH cells, expression of $M Y C N$ transcript and protein was not detected. Based on the above results, we decided to generate IMR-32 clones with PHLDA1 downregulation to gather more data on functions of the protein in our model of GD2-positive cells targeted with 14G2a mAb. Hence, GD2 negative SK-N-SH cells were not further investigated.

Silencing of PHLDA1 enhances ATP levels, mitochondrial membrane potential and decreases activity of caspases-3 and -7. Previously, we showed that PHLDAl expression is upregulated in IMR-32 neuroblastoma cells by treatment with the $14 \mathrm{G} 2 \mathrm{a}$ mAb (17). Thus, we aimed to verify roles of PHLDA1 in our model, as there is still controversy regarding functions of the protein in cancer cells. To address the issue, we decided to silence PHLDAl expression in $M Y C N$-amplified IMR-32 cells and verify effects of that on phenotype of the cell line. Although the PHLDA1 gene expression was not completely abolished, a significant decrease of PHLDAl expression was observed both on mRNA and protein levels in selected clones as compared to control (Mock1 and Mock2) and non-transduced cells (wild-type, WT, set here as control, equal to 1) (Fig. 2A and B). The expression of mRNA of PHLDAl was measured for S1, S2, S3, S4 clones and determined as $0.23( \pm 0.1), 0.16( \pm 0.1), 0.28( \pm 0.08), 0.12( \pm 0.06)$, respectively $(\mathrm{p}<0.01)$. Also, a significant decrease in PHLDA1 protein expression was noted for S1, S2, S4 clones (used for further experiments) as compared to Mock1 and non-transduced cells.

Next, we aimed to characterize behavior of clones with PHLDA1 downregulation in cell culture. We compared metabolic activity of S1, S2, S4 clones to control Mock1 and WT cells by measuring ATP levels in the cultured cells for up to 6 days (Fig. 2C). From the experiments, we concluded that from day 4 of culture a clearly visible increase in ATP levels can be measured for all three clones with a decreased expression of PHLDA1 ( $p<0.05$ for the S2 clone for the days 1-6; and for the S4 and S1 clones, the days 4-6, as compared to wild-type cells). By flow cytometry, we could not correlate the higher ATP levels in cell cultures of the clones S2 and S4 with increased cell proliferation (Table I). On the contrary, for S2 and S4 clones, we measured slightly higher (ca., 8-9\%, p<0.05) percentage of cells in G0/G1 phase for the clones with downregulation of PHLDA1 and lower number of cells in S and 
A

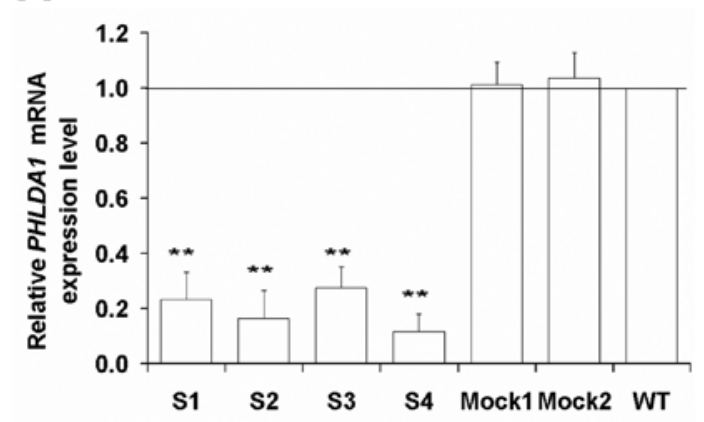

B

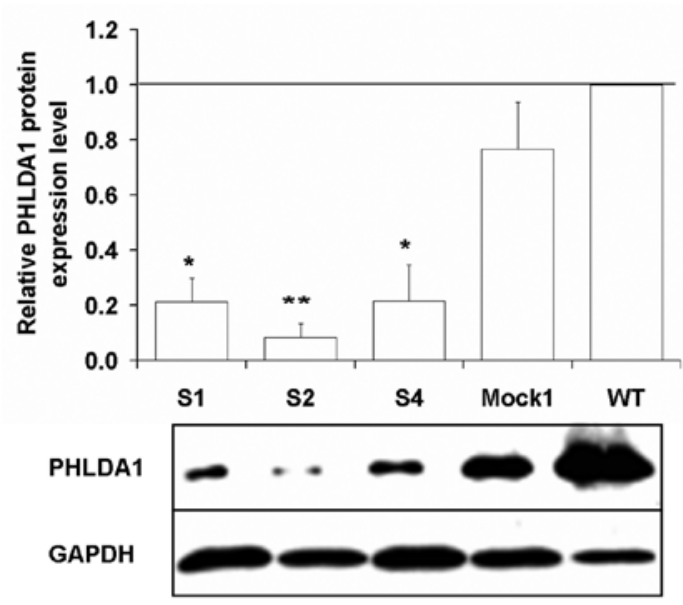

D

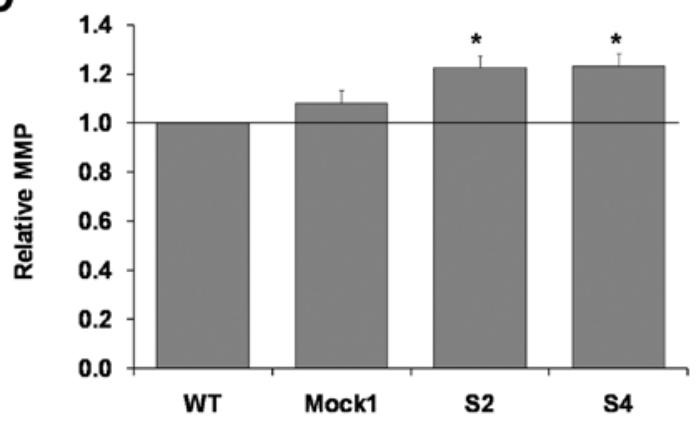

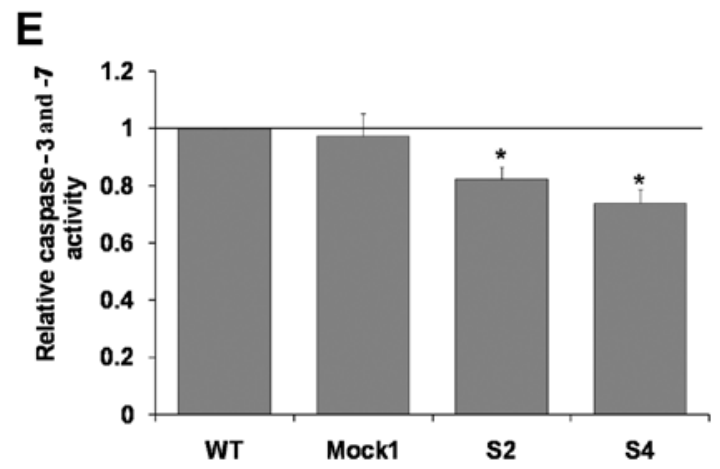

$\mathbf{F}$

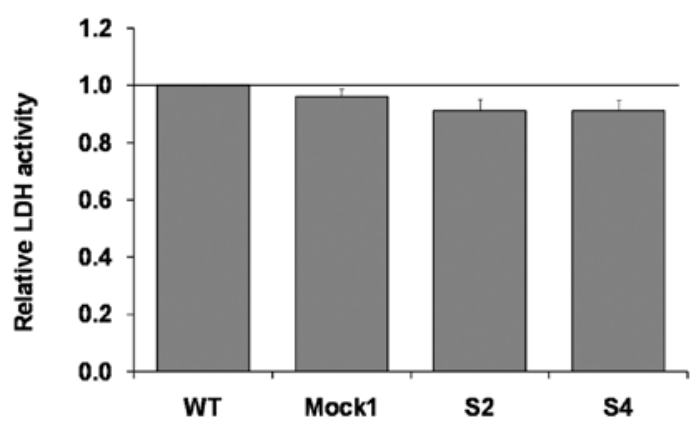

Figure 2. Characterization of clones of IMR-32 with stable silencing of PHLDA1 (S1-4), Mock1, Mock2, and WT cells. (A) mRNA levels of $P H L D A 1$ were measured by RT-qPCR in PHLDA1-silenced (S1, S2, S3, S4), Mock1, Mock2, and WT cells at $24 \mathrm{~h}$ after seeding of cells. $e E F-2$ cDNA was used as the reference. mRNA of $P H L D A 1$ in WT cells equals 1 . Data are presented as means of triplicates from three independent experiments $( \pm$ SEM) and calculated versus control values for WT cells, set as 1 (black baseline). (B) The PHLDA1 protein expression was measured at $48 \mathrm{~h}$ after seeding of cells in PHLDA1silenced (S1, S2, S4), Mock1 and WT cells at $48 \mathrm{~h}$ and normalized to GAPDH. Below the chart a representative immunoblot is presented. (C) Relative cellular ATP level was analyzed in PHLDA1-silenced (S1, S2, S4), Mock1 and WT cells. The y-axis shows the relative ATP level calculated as luminescent signal at specific time-point/luminescent signal at day 1 . Standard errors of the mean bars were omitted from the graph for clarity, but were $<0.9$ for all data points. (D) Levels of mitochondrial membrane potential (MMP) were measured using flow cytometry to detect accumulation of the MitoTracker Red CMXRos dye. Samples were run in duplicates. Relative activities of caspase-3 and -7 (E), LDH (F) were analyzed in PHLDA1-silenced (S2, S4), Mock1 and WT cells and calculated as caspase-3/7 or LDH activity at specific time-point/caspase-3 and -7 or LDH activity at day 1 . Means of three independent experiments $\left( \pm\right.$ SEM) are calculated versus control values for WT cells, set as 1 (black baseline). P-values for t-test were as follows: ${ }^{*} \mathrm{p}<0.05,{ }^{* *} \mathrm{p}<0.01$.

G2/M phases, as compared to WT cells. Interestingly, we also measured lower numbers of apoptotic cells in the S2 and S4 cells. Such findings prompted us to analyze MMP, activity of apoptosis-executing caspase- 3 and -7 in cells, and levels of LDH released to culture media (Fig. 2D-F). We measured ca. $20 \%$ higher intensity of fluorescence due to accumulation of the dye MitoTracker Red CMXRos $(\mathrm{p}<0.05)$, which was used for analysis of mitochondrial membrane potential in the cells with partial PHLDAl silencing, as compared to WT cells (set as 1). Also, we report that the clones S2 and S4 showed lower activity of the caspases (on the day 6 of culture, $p<0.05$ ). LDH leakage was slightly and insignificantly decreased in the S2 and S4 clones. To summarize, downregulation of PHLDA1 in IMR-32 cells can be linked to firm changes in cell 
A

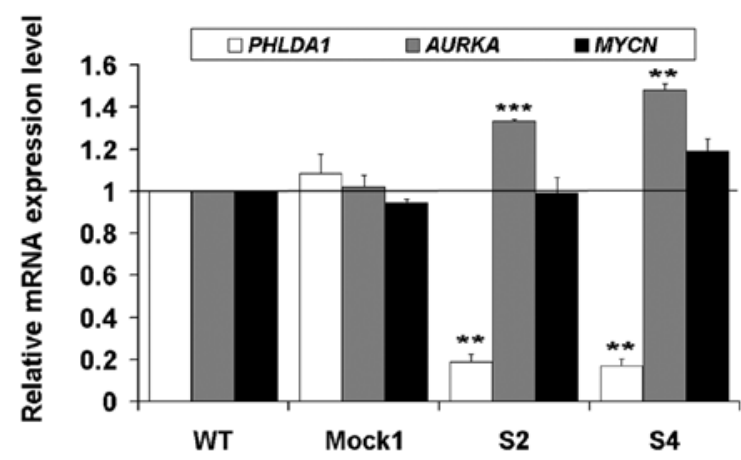

C

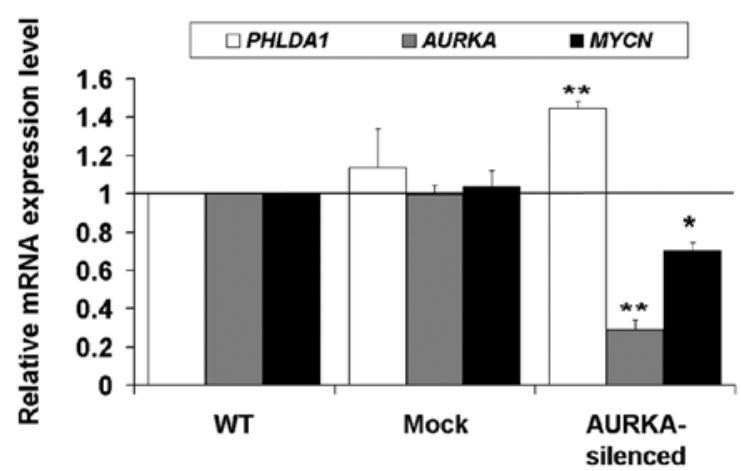

E
B

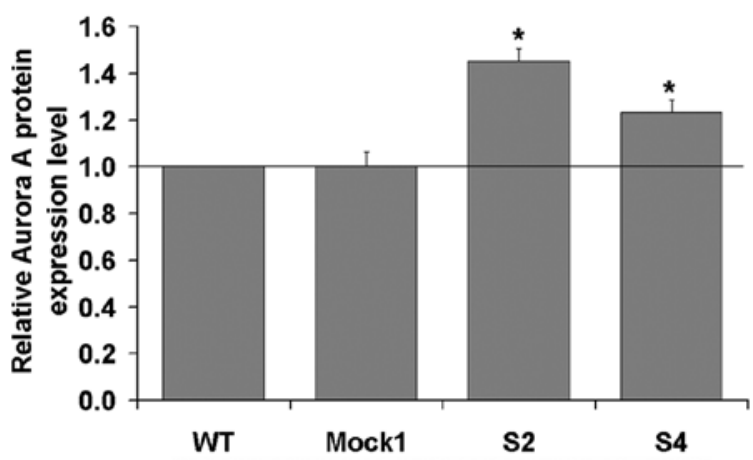

Aurora A a-tubulin

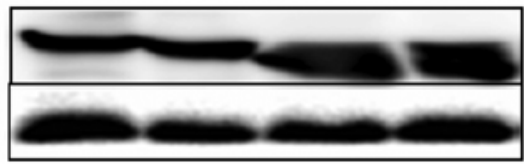

D

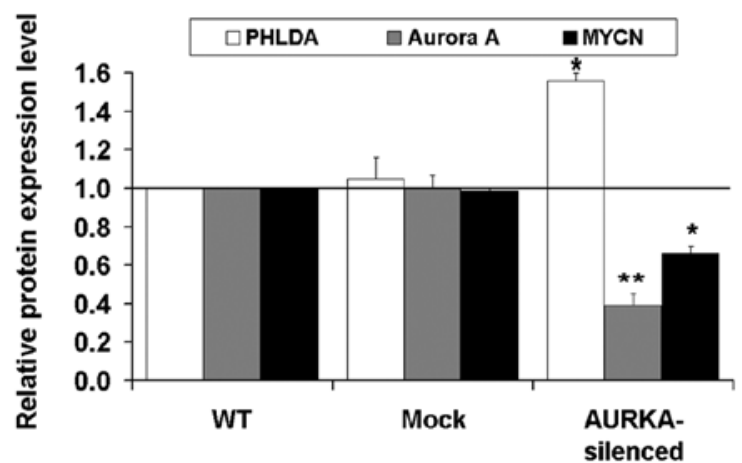

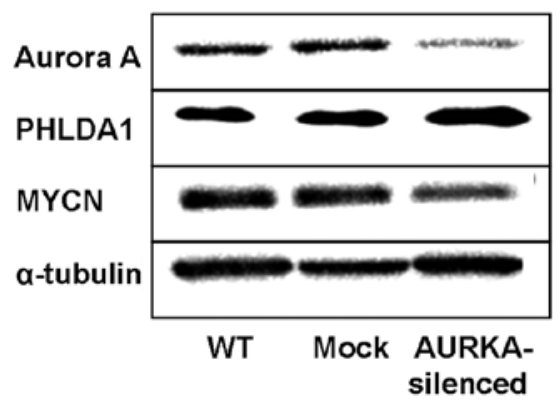

Figure 3. The expression of mRNA and protein of the PHLDA1, AURKA and MYCN genes in PHLDA1-stably silenced, Mock1 and WT IMR-32 cells and in AURKA-transiently silenced, Mock and WT IMR-32 cells. (A) mRNA levels of PHLDA1, AURKA and MYCN were measured by RT-qPCR in PHLDA1-silenced, Mock1 and WT cells. RPS13 cDNA was used as the reference. (B) Aurora A protein expression was measured in PHLDA1-silenced (S2, S4), Mock1 and WT cells at $48 \mathrm{~h}$ after seeding and normalized to $\alpha$-tubulin. Below the chart a representative immunoblot is presented. (C) mRNA levels of PHLDAl, AURKA and MYCN were measured in AURKA-silenced, Mock and WT cells. $e E F-2$ cDNA was used as the reference. AURKA, MYCN and PHLDA1 expression in wild-type cells equals 1 (black baseline). (D) Expression levels of the Aurora A, PHLDA1 and MYCN proteins were measured by western blot analysis and normalized to $\alpha$-tubulin. Data are presented as means of triplicates from three independent experiments ( \pm SEM). P-values for t-test were as follows: ${ }^{*} p<0.05,{ }^{* *} \mathrm{p}<0.01,{ }^{* * *} \mathrm{p}<0.001$. (E) Representative immunoblots are presented as a separate section of the figure.

phenotypes and to some extent explain the higher ATP levels measured for the S2 and S4 clones.

Characterization of expression of selected genes involved in neuroblastoma cell fate. Aurora A is an important oncogenic driver overexpressed in majority of high risk neuroblastomas (34). In MDA-MB-231 breast cancer cells, Aurora A is a partner of PHLDA1 and more importantly both proteins are engaged in mutual regulation (10). Additionally, among several substrates, Aurora A complexes MYCN and stabilizes the transcription factor by preventing it from Fbxw7-driven proteasomal degradation (11). Hence, we decided to analyze the expression of the AURKA and MYCN genes in PHLDAIsilenced clones: S2 and S4 (Fig. 3A). We observed that in the 
A

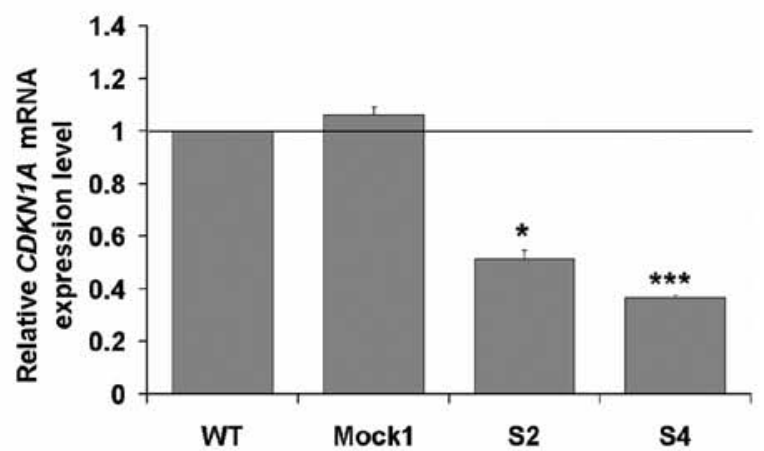

C

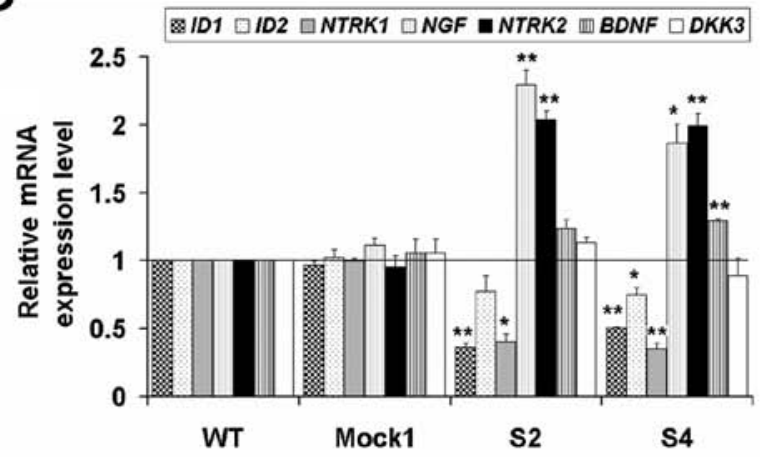

B

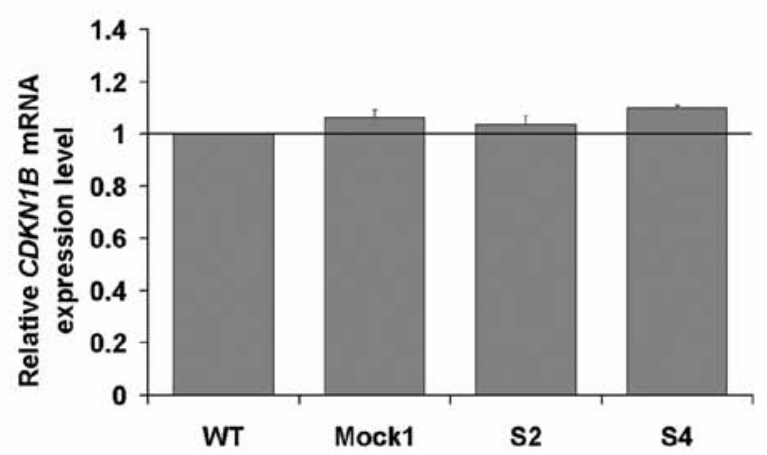

D

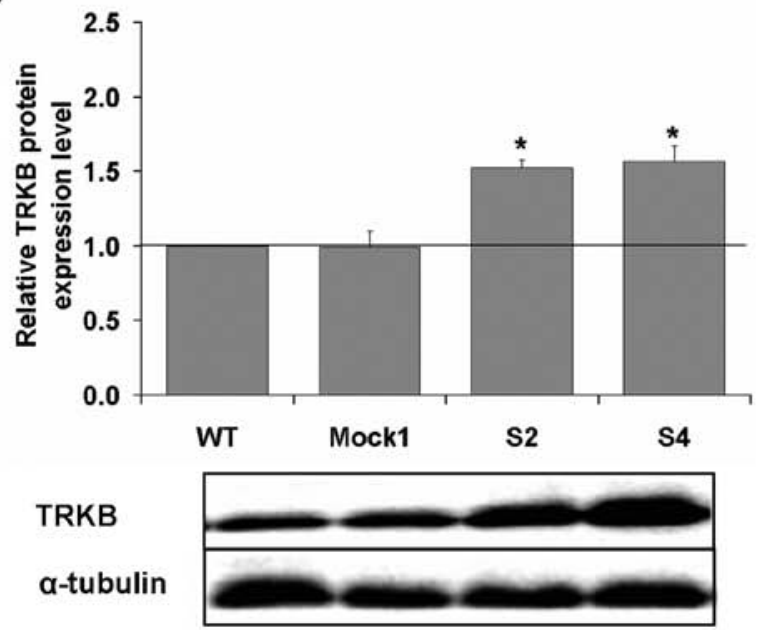

Figure 4. Expression levels of the $C D K N 1 A$ and $C D K N 1 B$ genes and selected markers of differentiation in PHLDAl-silenced, Mock1 and WT IMR-32 cells. Expression of CDKN1A (A) and CDKN1B (B) genes was measured by RT-qPCR in PHLDA1-silenced, Mock1 and WT cells. (C) Gene expression levels of differentiation markers: ID1, ID2, NTRK1, NGF, NTRK2, BDNF, DKK3 were measured by RT-qPCR. RPS13 cDNA was used as the reference. Gene expression in WT cells equals 1 (black baseline). (D) TRKB protein expression was measured in PHLDAl-silenced (S2, S4), Mock1 and WT cells at $48 \mathrm{~h}$ after seeding and normalized to $\alpha$-tubulin. Below the chart a representative immunoblot is presented. Data are presented as means of triplicates from three independent experiments $\left( \pm\right.$ SEM). P-values for t-test were as follows: ${ }^{*} \mathrm{p}<0.05,{ }^{* *} \mathrm{p}<0.01,{ }^{* * *} \mathrm{p}<0.001$

PHLDA1-silenced clones, expression of mRNA of AURKA was statistically significantly induced to $1.33 \pm 0.01$ in $\mathrm{S} 2$ and to $1.48 \pm 0.03$ in $\mathrm{S} 4$, as compared to non-transduced cells (set as 1 ). Moreover, we measured a statistically meaningful increase in Aurora A levels in the clones S2 and S4 (Fig. 3B, p<0.05). However, MYCN expression was unchanged in the clone S2 and increased ca. 20\% in the clone S4 (but without statistical significance) (Fig. 3A). This further prompted us to check the effect of silencing of Aurora A on expression of its two partners, i.e., PHLDA1 and MYCN. As AURKA expression is crucial for cell mitosis, we were unable to select single clones of IMR-32 cells with the gene knockout, due to their poor proliferation (data not shown). However, in pools of IMR-32 cells, collected 4 days after lentiviral transduction, there was a statistically significant decrease in both mRNA and protein levels of $A U R K A$, along with a meaningful increase in levels of mRNA and protein of PHLDAl and a decrease of mRNA and protein of $M Y C N$ (Fig. 3C-E). Also, as previously reported by others (11), we can confirm that in AURKA-knockout experiments, the MYCN level was decreased along with silencing of expression of AURKA.
We extended the above findings with analyses by RT-qPCR of mRNA levels of genes important for cell cycle and differentiation, such as $C D K N 1 A$ (encoding for p21), $C D K N 1 B$ (encoding for p27), ID1, ID2, NTRK1 (encoding TRKA), NTRK2 (encoding TRKB), BDNF (encoding one of the two main ligands of TRKB), $N G F$ (encoding the main ligand of TRKA) and DKK3 in PHLDAl-silenced clones (as compared to controls, set as 1) (Fig. 4). The transcript of CDKNIA was decreased, but no changes for the transcript of $C D K N 1 B$ were observed (Fig. 4A and B). We report meaningfully significant decrease of IDI (for S2 and S4, p<0.01) and ID2 expression (for $\mathrm{S} 4, \mathrm{p}<0.05$ ). An inverse correlation between transcripts of NTRKI and NGF was measured. Thus, expression of NTRK1 was decreased ca. 4-fold $(\mathrm{p}<0.05)$ and expression of $N G F$ was increased ca. 2-fold $(\mathrm{p}<0.05)$. Also, expression of NTRK2 was advanced ca. 2-fold ( $\mathrm{p}<0.01)$, while expression of one of the genes encoding for its ligand, $B D N F$, was only slightly increased. The transcript of $D K K 3$ was not statistically significantly affected (Fig. 4C). Finally, we measured a statistically meaningful increase in TRKB levels in the clones S2 and S4 (Fig. 4D, p $<0.05$ ). The data show that PHLDA1 downregula- 
A

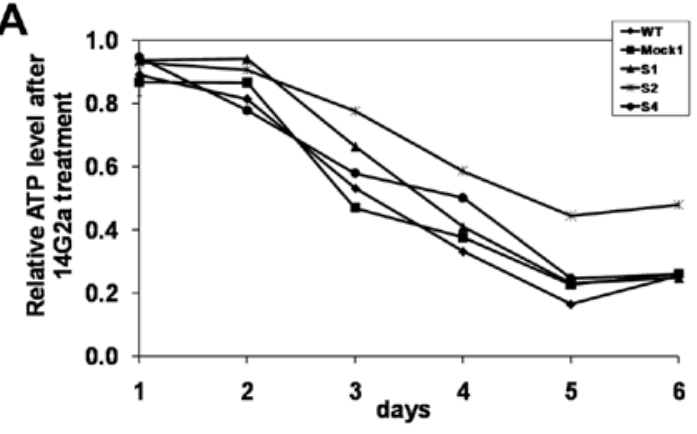

C
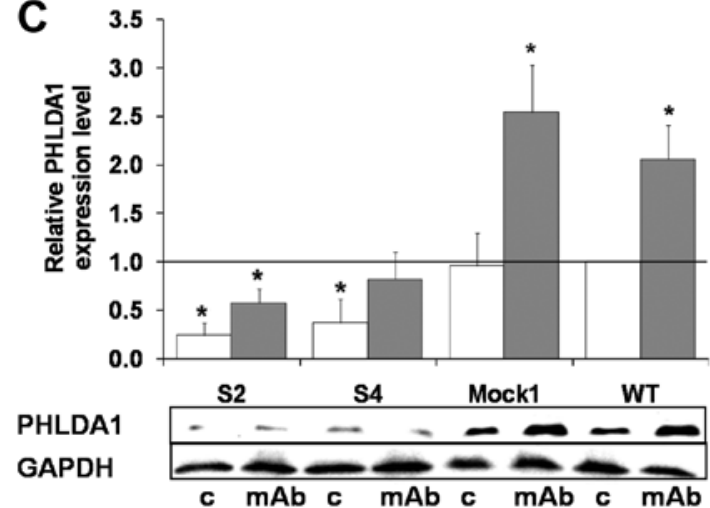

$\mathbf{E}$

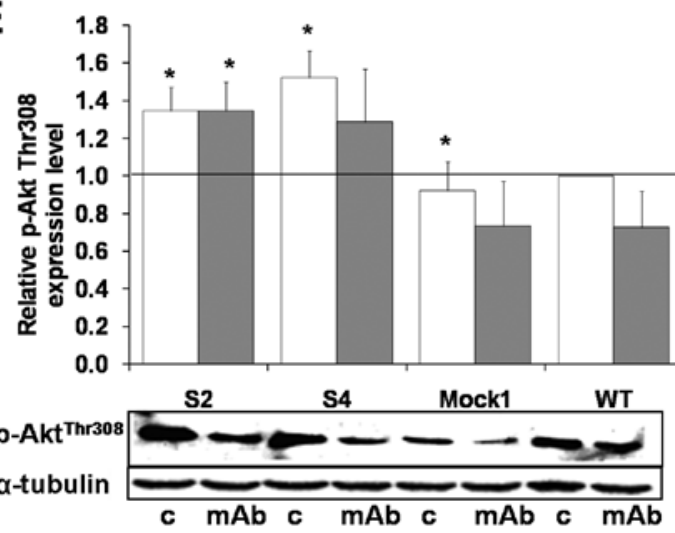

G

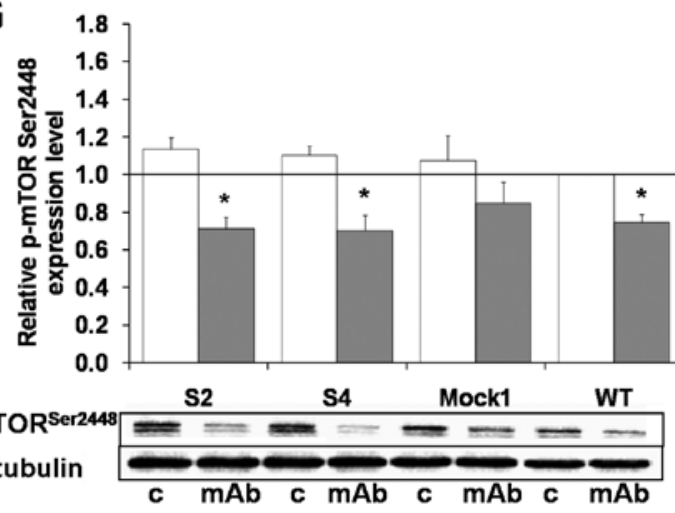

B 1.8
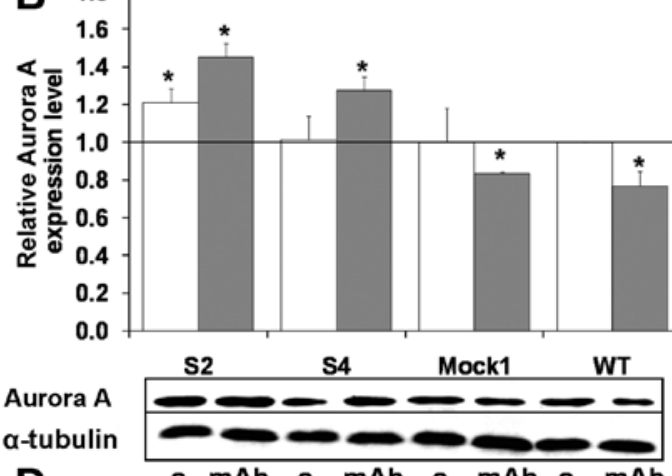

D 1.8

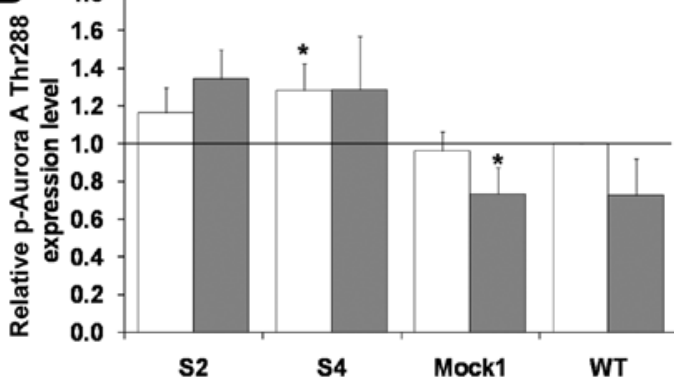

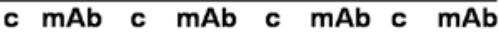
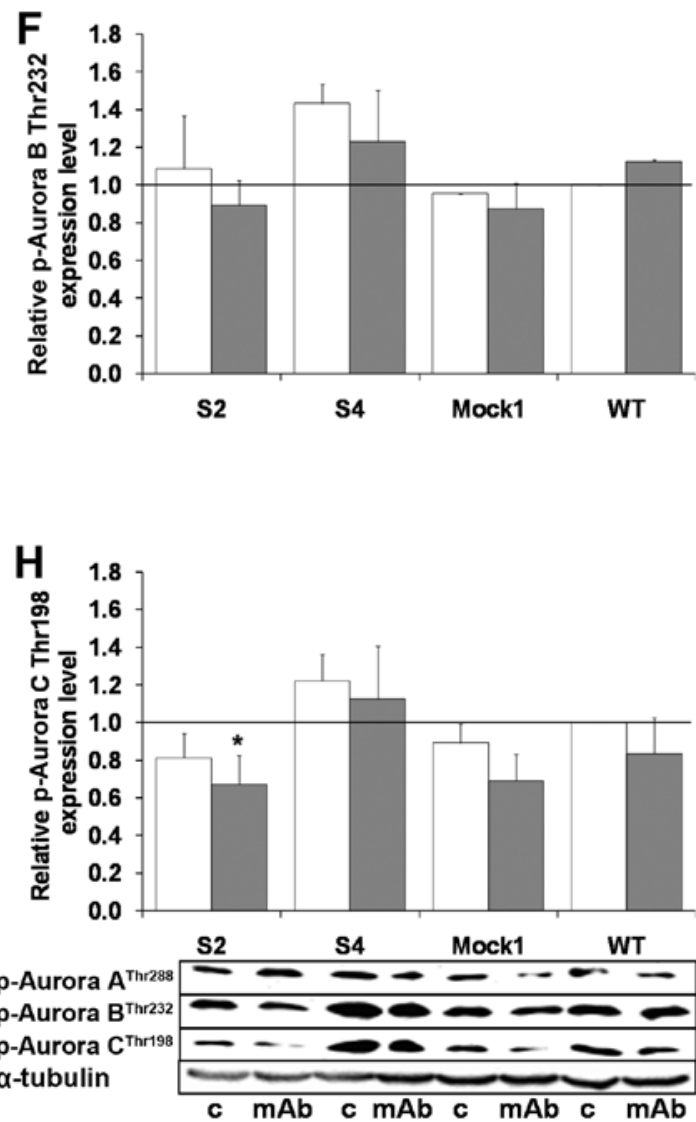

Figure 5. Effects of the 14G2a mAb on cellular ATP, levels of PHLDA1, Aurora A, selected activating phosphorylations of aurora kinases, Akt, mTOR in PHLDA1-silenced, Mock1 and WT cells. (A) Relative cellular ATP levels were analyzed in PHLDA1-silenced (S1, S2, S4), Mock1 and WT cells. The y-axis shows the relative ATP level calculated as luminescent signal at specific time-point/luminescent signal at day 1. Means of three independent experiments $( \pm$ SEM) are calculated. The relative ATP level of particular type of cells after the $14 \mathrm{G} 2 \mathrm{a}$ mAb treatment was calculated versus control values - untreated cells (black baseline at the top of the chart), set as 1 . Standard errors of the mean were omitted from the graph for clarity, but were $<0.4$ for all data points. Aurora A (B) and PHLDA1 (C) expression levels as well as phosphorylation of Aurora A [(D) p-Aurora A, Thr288], Aurora B [(F) p-Aurora B, Thr232], Aurora C [(H) p-Aurora C, Thr198], Akt [(E) p-Akt, Thr308] and mTOR [(G) p-mTOR, Ser2448] kinases were measured at 48 h in 14G2a-treated and control cells. The proteins were normalized to GAPDH (for PHLDA1) or $\alpha$-tubulin (for the rest of proteins). Representative immunoblots are presented below the charts in (B, C, E, G and H): c, control cells; mAb, mAb-treated cells $(40 \mu \mathrm{g} / \mathrm{ml})$. Mean values of three separate experiments $( \pm$ SEM) obtained for the $14 \mathrm{G} 2 \mathrm{a}$ mAb-treated IMR-32 are presented (grey bars) and calculated versus control values (white bars). Expression levels of individual proteins in WT IMR-32 cells were used as the reference (black baseline, set as 1). P-value for t-test was: " $\mathrm{p}<0.05$. 
A

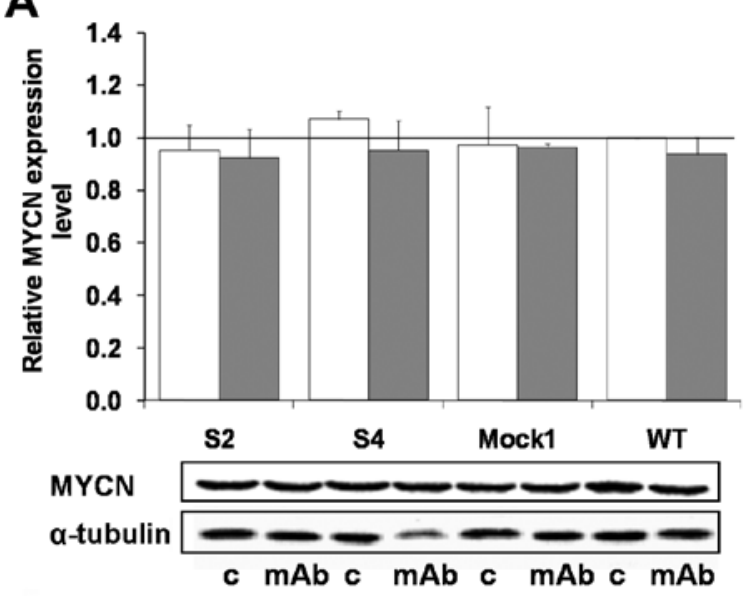

C

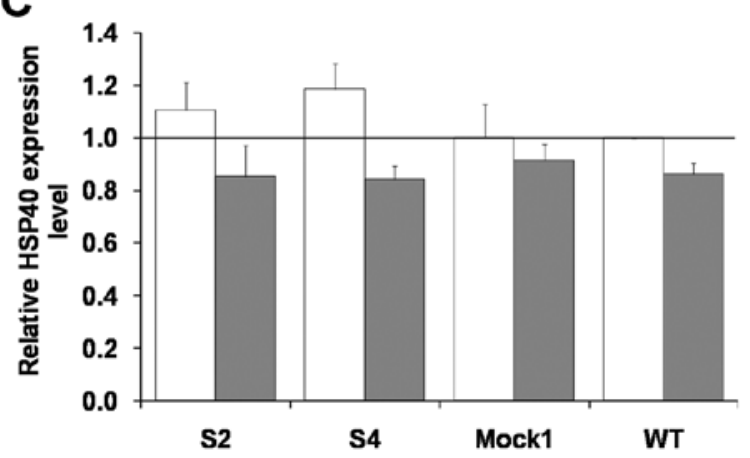

B

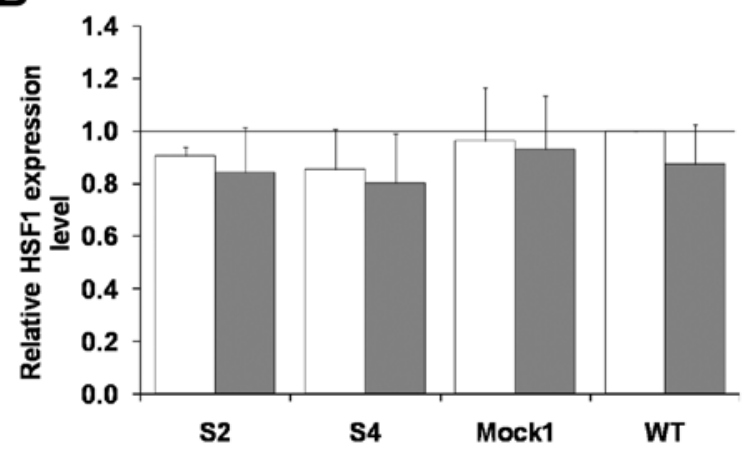

D

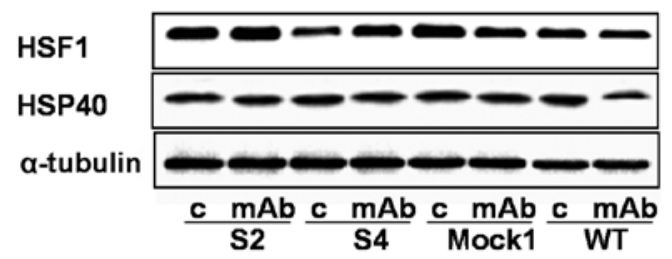

Figure 6. Effects of the 14G2a mAb on MYCN, HSF1, HSP40 expression in PHLDA1-silenced, Mock1 and WT IMR-32 cells. MYCN (A), HSF1 (B) and HSP40 (C) proteins expression was measured at $48 \mathrm{~h}$ and normalized to $\alpha$-tubulin. Representative immunoblots are presented in (A and D): c, control cells; $\mathrm{mAb}, \mathrm{mAb}$-treated cells $(40 \mu \mathrm{g} / \mathrm{ml})$. Mean values of three separate experiments $( \pm \mathrm{SEM})$ obtained for the $14 \mathrm{G} 2 \mathrm{a}$ mAb-treated IMR-32 are presented (grey bars) and calculated versus control values (white bars). Expression level of proteins in WT IMR-32 cells was used as the reference (black baseline, set as 1).

tion affects transcripts of some of the aforementioned genes involved in cell cycle and differentiation. Also, levels of TRKB were raised in the clones with PHLDA1-downregulation. Levels of p21 and p27 were investigated in the clones S2, S4, Mock1 and WT in both 14G2a mAb-treated and untreated cells (as can be seen in more detail in 'PHLDAl downregulation affects apoptosis- and autophagy-related proteins in IMR-32 cells' and in Fig. 8A and B). However, to draw further conclusions levels of remaining proteins should be investigated.

PHLDAl downregulation increases phosphorylation of Aurora A and Akt kinases. Previously, we reported that IMR-32 cells are sensitive to direct cytotoxicity of $14 \mathrm{G} 2 \mathrm{a}$ mAb with partial involvement of apoptosis (18). Hence, using the model, we tested if the knockout of PHLDAl expression will affect sensitivity of the clones to treatment with the $14 \mathrm{G} 2 \mathrm{a}$ mAb (40 $\mu \mathrm{g} / \mathrm{ml}$ ). First, we analyzed expression of GD2 ganglioside on the clones $\mathrm{S} 2$ and $\mathrm{S} 4$ by flow cytometry and concluded that the glycolipid level was unchanged as compared to controls (data not shown). Next, we measured ATP levels of clones treated with 14G2a for up to 6 days (Fig. 5A) and we report that the clone $\mathrm{S} 2$ was the most refractory to incubation with $14 \mathrm{G} 2 \mathrm{a}$ from the three clones and controls tested $(\mathrm{p}<0.05$ for the $\mathrm{S} 2$ clone, days 1-6; for the $\mathrm{S} 4$ clone, day 4; and the $\mathrm{S} 1$ clone, days 2-3; as compared to wild-type cells).
Our earlier studies showed that treatment of IMR-32 cells with 14G2a led to increase in PHLDA1 levels in IMR-32 cells, accompanied by a decrease in: total levels of MYCN (25), Aurora A and B, activating phosphorylations of Aurora A (Thr288), Aurora B (Thr232), Aurora C (Thr198) (17), Akt (Thr308), and mTOR (Ser2448) in whole cell extracts (24). Thus, we compared the levels of the aforementioned proteins between the S2 and S4 clones and control cells after $48 \mathrm{~h}$ of the 14G2a-treatment (Figs. 5B-H and 6A). Since PHLDA1 levels were detectable in the clones $\mathrm{S} 2$ and $\mathrm{S} 4$, we could also measure an increase in the protein levels, as a result of addition of 14G2a (Fig. 5C). Moreover, we report that concomitantly with the highest resistance to treatment with $14 \mathrm{G} 2 \mathrm{a}$ of the S4 clone, the level of phosphorylated Aurora A (Thr288) was the highest in both treated and untreated cells among all tested samples. Similar effects can be observed for the S2 clone (Fig. 5D and the blot below $\mathrm{H}$ ). Interestingly, in the $\mathrm{S} 4$ clone the levels of phosphorylated Aurora B (Thr232) and Aurora C (Thr198) were also elevated (Fig. 5F and H). Finally, levels of phosphorylated Akt (Thr308) were the highest in the clones S2 and S4 (Fig. 5E). Strikingly, after the $14 \mathrm{G} 2 \mathrm{a}-\mathrm{mAb}$ treatment, clearly opposite directions of the changes of Aurora A, p-Aurora A (Thr288), p-Akt (Thr308) were observed in PHLDA1-downregulated cells when compared to control cells (Fig. 5B, D and E and the blot below $\mathrm{H}$ ). Thus, for the $\mathrm{S} 2$ and $\mathrm{S} 4$ clones the levels of the 
A

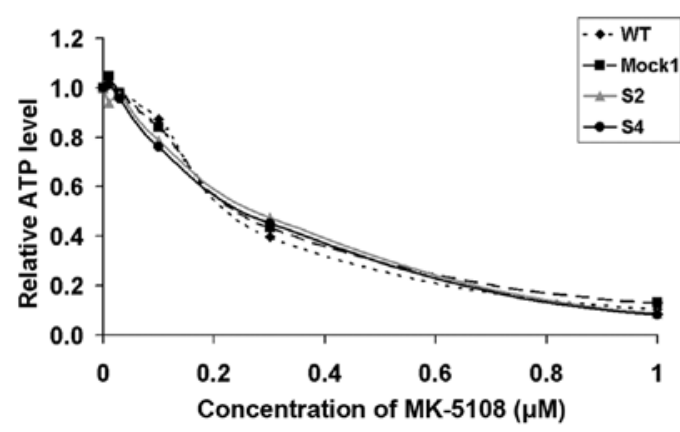

C

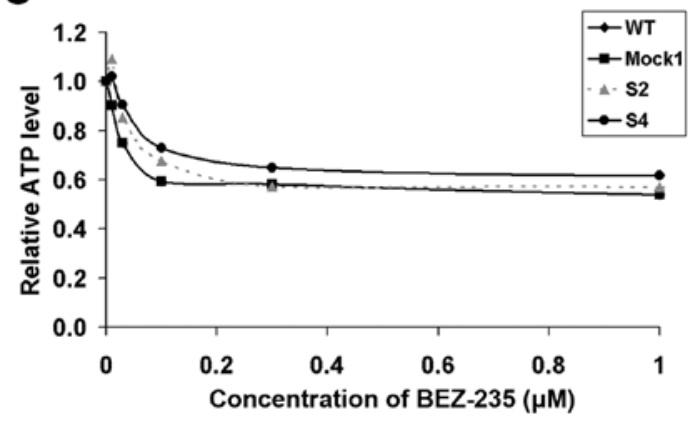

B

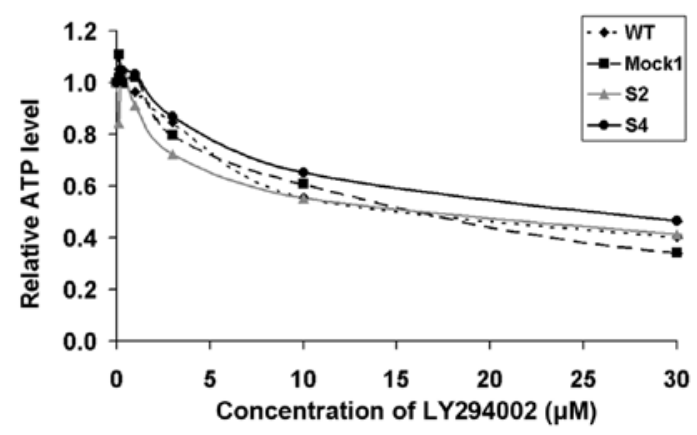

Figure 7. Susceptibility of PHLDA1-silenced clones, Mock1, WT cells of IMR-32 to MK-5108, LY294002 and BEZ-235 inhibitors. Cells were incubated with Aurora A kinase inhibitor [MK-5108 (A)], and PI3K and mTOR dual inhibitors [LY294002 (B) and BEZ-235 (C)]. Inhibitors were added to cell cultures for $72 \mathrm{~h}$ and relative ATP levels were measured. Control cells were treated with equivalent volume of DMSO or water (solvents for the inhibitors). Mean values of three separate experiments ( \pm SEM) are shown. proteins were increased, but for Mock1 and wild-type clones the levels were decreased. There were no changes in phosphorylated mTOR (Ser2448) kinase expression levels between PHLDA1silenced clones, control and non-transduced cells (Fig. 5G). No statistically significant changes in MYCN, HSF1, HSP40 expression was observed for the cell populations tested, either in the $14 \mathrm{G} 2 \mathrm{a}$ mAb treated and untreated IMR-32 cells (Fig. 6).

Aurora A and Akt are binding partners. Moreover, Aurora A overexpression was reported responsible for induction of chemoresistance via Akt phosphorylation of Ser473 (35). Thus, we checked whether the reduction of levels of PHLDA1 and the accompanying rise of levels of p-Aurora A (Thr288) and p-Akt (Thr308) would translate into a higher resistance to selected inhibitors, i.e., MK-5108 (Aurora A inhibitor), LY294002 and BEZ-235 (dual PI3K and mTOR inhibitors). We report that susceptibility of PHLDA1-silenced clones to aforementioned inhibitors remained unchanged as compared to controls (Fig. 7). To conclude, we could observe that effects of the 14G2a mAb addition on ATP levels of tested cells were dependent on PHLDA1 levels in IMR-32 cells. This confirms our previously reported observation that PHLDA1 is linked to treatment with the $14 \mathrm{G} 2 \mathrm{a}$ mAb. However, no such correlations were found for the inhibitors used.

PHLDA1 downregulation affects apoptosis- and autophagyrelated proteins in IMR-32 cells. Previously we reported that incubation of IMR-32 cells with 14G2a led to induction of apoptosis (18). Also the cytotoxicity of the mAb was linked to a meaningful rise of levels of p53 (17). To extend the findings, we analyzed changes of p21, p27, p53, cleaved PARP and an apoptosis-executing caspase-3 in the clones S2 and S4, as compared to controls (Fig. 8). We report that p21 did not change in the $\mathrm{mAb}$ or the control cells of any of the clones, p27 decreased in the untreated S2 and S4 cell clones, and p53 increased to similar levels in all $\mathrm{mAb}$-treated populations tested (Fig. 8A-C and H). Levels of caspase-3 and PARP were lower in the S2 and S4 clones. Moreover, although an increase in cleaved caspase-3 and PARP was measured for all cells treated with the $14 \mathrm{G} 2 \mathrm{a}$ mAb, the levels of the proteins were lower in the S2 and S4 samples, when compared to respective controls (WT, set as 1 , Fig. $8 \mathrm{E}, \mathrm{G}$ and $\mathrm{H}$ ). On the contrary, we measured a rise in levels of an autophagy-related protein, converted LC3A/B-II form in the S2 and S4 clones, as compared to respective controls of both $14 \mathrm{G} 2 \mathrm{a}$-treated and untreated groups (Fig. 8D and H). This was accompanied by similar levels of Atg7 in all samples tested (Fig. 8F and $\mathrm{H}$ ).

The data suggest that downregulation of PHLDA1 in our model may make the cells less prone to apoptosis and positively regulate autophagosome formation.

\section{Discussion}

The goal of our research was to characterize IMR-32 cells with a downregulation of PHLDA1. We were able to show several differences in expression of transcripts and proteins of genes important for neuroblastoma survival. Hence, in both S2, S4 clones with decreased PHLDA1 levels, we found increased mRNA and protein levels of AURKA and NTRK2 [Aurora A and TRKB are markers of poor prognosis $(34,36)]$, but a decrease in mRNA of NTRK1 [TRKA is a marker of good prognosis (36)]. NTRK1 and NTRK2 encode key proteins involved in regulation of growth arrest and differentiation of neuroblastomas. In the future, the above findings should be extended with analyses of levels of proteins of TRKA and TRKB and their ligands, also with more insights into possible isoforms of the TRK receptors that result from alterantive splicing and have differential influence on cell behaviour $(37,38)$. 
A

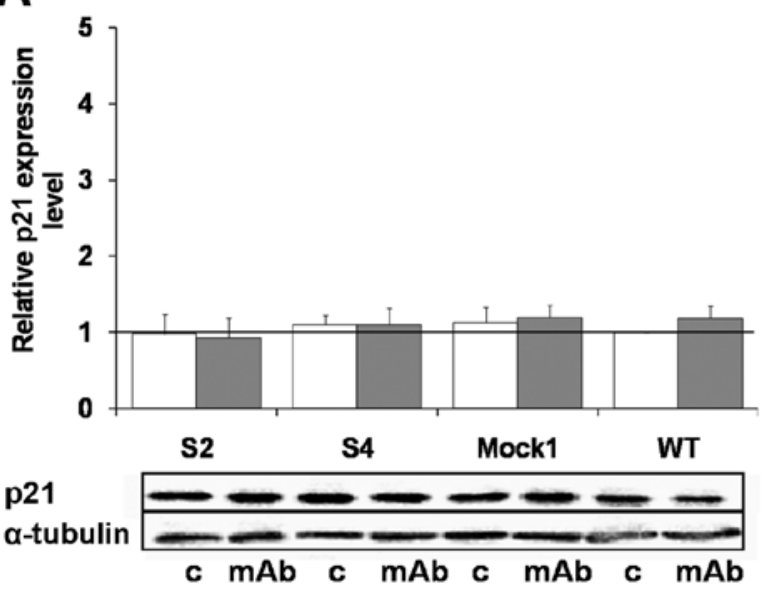

C

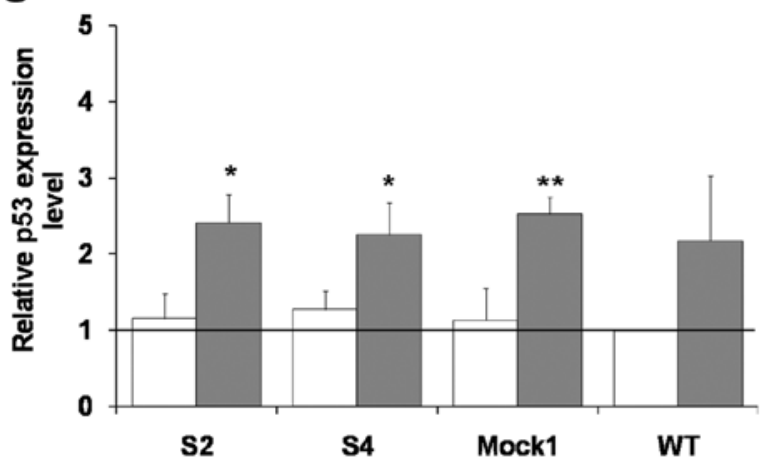

E

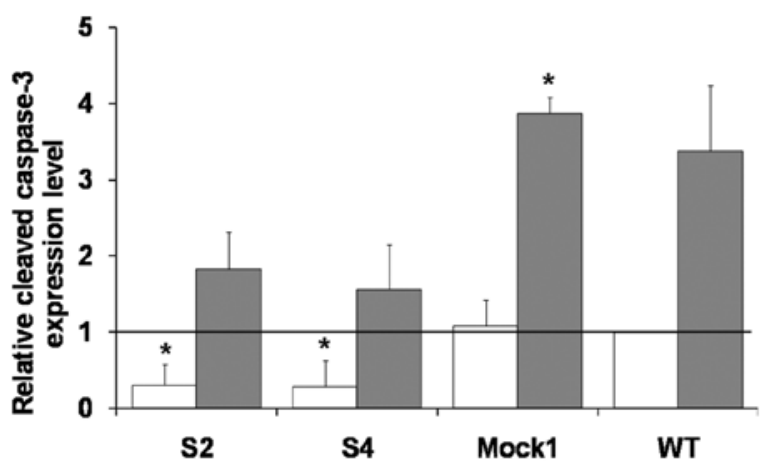

G

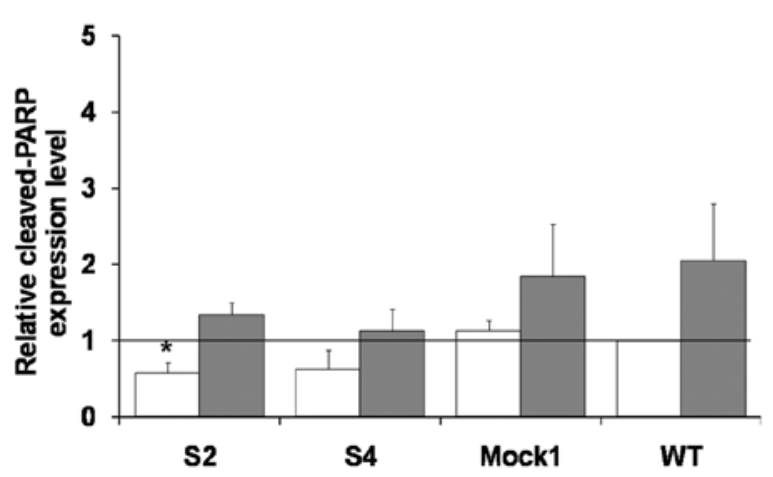

B

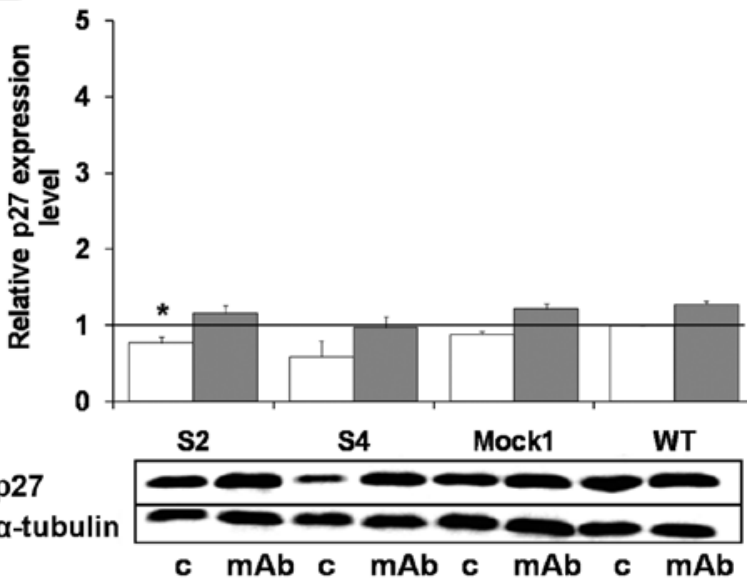

D

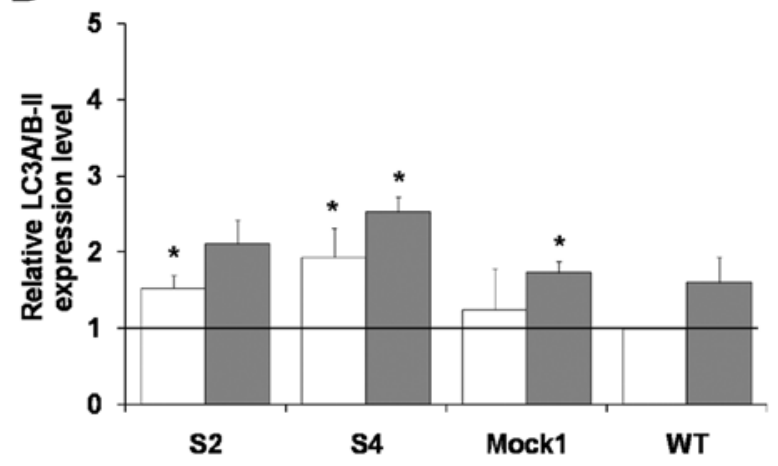

$\mathbf{F}$

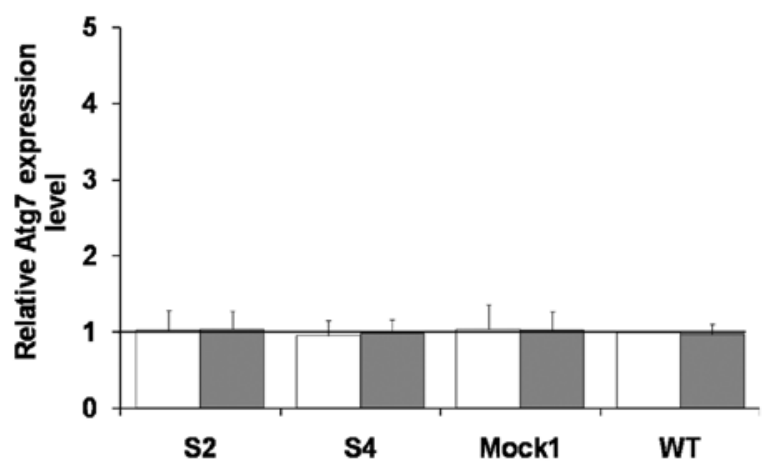

H

p53

cleaved

caspase-3

cleaved

PARP

LC3A/B-I

LC3A/B-II

Atg7

GAPDH

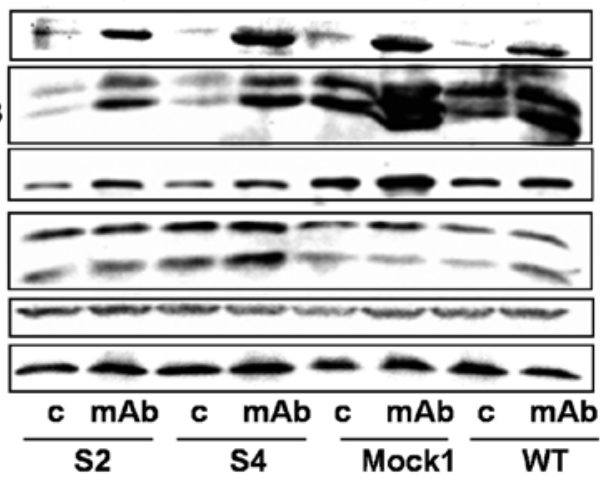

Figure 8. Effects of the 14G2a mAb on expression of apoptosis- and autophagy-associated proteins in PHLDA1-silenced, Mock1 and WT cells. Expression levels of p21 (A), p27 (B), p53 (C), LC3A/B-II (D), cleaved caspase-3 (E), cleaved PARP (G), and Atg7 (F) were measured at $48 \mathrm{~h}$ and normalized to $\alpha$-tubulin (for p21 and p27) or GAPDH (for the rest of the proteins). Representative immunoblots are presented below A and B charts and in a separate section of the figure $(\mathrm{H})$ : c, control cells; mAb, mAb-treated cells $(40 \mu \mathrm{g} / \mathrm{ml})$. Mean values of three separate experiments $( \pm \mathrm{SEM})$ obtained for the 14G2a mAb-treated IMR-32 are presented (grey bars) and calculated versus control values (white bars). Expression level of proteins in WT IMR-32 cells was used as the reference (black baseline, set as 1). P-values for t-test were as follows: ${ }^{*} \mathrm{p}<0.05,{ }^{* *} \mathrm{p}<0.01,{ }^{* * * *} \mathrm{p}<0.001$. 
Oncogenic functions of Aurora A in neuroblastoma are well established (11). Akt and Aurora A are partners and important targets for neuroblastoma treatment $(13,39)$. Akt was shown to transduce signal to chemorestistance induced via TRKB/BDNF (40) and to participate in signaling network from Aurora A that drives tumor growth (35). Also, Akt is able to phosphorylate tumor suppressors p21 and p27, both on two sites, i.e., at Thr145 and Ser153 in p21 and at Thr157 and Thr198 in p27, thus leading to cytoplasmic retention of the proteins $(41,42)$. On the protein level, we observed an increase in Aurora A, activating phosphorylations of p-Aurora A (Thr288), p-Akt (Thr308), and a decrease in p27 (for the clones S2 and S4). Together with the aforementioned changes of expression of NTRK1 and NTRK2, our data suggest that downregulation of PHLDA1 in IMR-32 cells may affect regulation of cell growth and/or survival. However, more research is needed to elucidate how silencing of PHLDA1 expression may be linked to mechanism by which Aurora A and Akt promote tumorigenesis, as an inverse correlation in expression of PHLDA1 and selected phosphorylation of Aurora A and Akt was observed in our model. Another group of our findings stems from analyses of levels of PHLDA1 in IMR-32 cells with downregulation of $A U R K A$, where again such an inverse correlation between expression of PHLDA1 and Aurora A was confirmed. Also, in the most of the analyzed neuroblastoma cell lines, i.e., IMR-32, SK-N-SH, Kelly, CHP-134, HTLA230 such inverse correlations can be stated. Hence, the above findings allow us to hypothesize that in IMR-32 cells Aurora A and PHLDA1 are proteins that might negatively regulate each other, as reported previously by Johnson et al for the MDA-MB-231 breast cancer cells (10). Therefore, further experiments should be performed to show a direct physical interactions of the proteins. Also, experiments with additional cell lines or approches aiming to overexpress PHLDA1 in cell lines such as HTLA230, Kelly, and CHP-134 could add more data on the roles of the protein in neuroblastoma cells.

Previously, we reported that the increased level of PHLDA1 in IMR-32 cells treated with $14 \mathrm{G} 2 \mathrm{a}$ mAb correlated with the decreased level of Aurora A, p-Aurora A (Thr288) and p-Akt (Thr308) (17,24). Also, a specific Aurora A inhibitor, MK-5108, affected the PHLDA1 level in a time-dependent manner in the cell line (17). As expected, clones of IMR-32 cells with downregulation of PHLDAl were less sensitive to treatment with the $14 \mathrm{G} 2 \mathrm{a}$ mAb. However, higher levels of activating phosphorylations of Aurora A and Akt could not be correlated with higher resistance to treatment with Aurora A inhibitor (MK-5108), and two dual PI3K and mTOR inhibitors (BEZ-235 and LY294002). As the results of cytototoxicity experiments were determined based on cellular ATP measurements we cannot exclude that differences in ATP levels of given cell clones can affect the results. In the future, the findings should be confirmed with other methods.

We reported effects of PHLDAI silencing in IMR-32 cells on some features of mitochondria. Hence, we measured an increase in mitochondrial membrane potential and enhanced cellular ATP levels, as compared to control cells. Because MMP is the driving force for mitochondrial ATP synthesis, gain of MMP may result in increased ATP level (43). However, to gain more information on possible changes of mitochondria in the cells, further tests should be performed including appli- cation of other dyes (e.g., JC-1) to confirm MMP changes as well as analyses of morphology and density of the organelles.

In our model, the changes of levels of ATP were not correlated with higher proliferation of the clones, but rather lower number of apoptotic cells in untreated cell cultures, decreased levels of cleaved caspase-3 and PARP, and decreased activity of caspase-3/7. This sugesst that downregulation of PHLDA1 expression may contribute to apoptosis resistance in our model. Such data support pro-apoptotic functions of PHLDA1 in IMR-32 cells and suggest a possible role of the protein in regulation of functions of mitochondria and/or mitochondrialmediated apoptotic pathways. Application of other than 14G2a inducers of apoptosis could help to further substantiate our conclusions.

Interestingly, we also found a correlation between decreased expression of PHLDA1 and increased levels of the autophagy marker, LC3A/B-II. From our data, a negative correlation between expression of PHLDA1 and LC3A/B-II in S2 and S4 clones suggests that PHLDA1 seems to be limiting autophagosome formation. Further studies are needed to determine if the observed rise of expression of LC3A/B-II results in advanced autophagy or a block of autophagolysomal maturation by analyzing the autophagic flux (44).

Links between mitochondria, apoptosis and autophagy are well known. Hence, mitophagy (a mitochondria specific autophagy) operates to remove demaged or excessive mitochondria in cells (45). Roles of mitochondrial dynamic proteins such as MFN 1/2 (mitofusin 1 and 2), OPA1 (optic athrophy 1), DRP1 (dynamin related protein 1), and PINK1 (PTEN-induced kinase 1)/Parkin in the two cell processes were previously described $(45,46)$.

Our study links PHLDA1 and some proteins involved in apoptosis and autophagy. However, based on the already published reports, the exact funtions of the protein seem to be dependent on a cell type tested and agents used to affect the PHLDA1 level. In the future, we plan to perform more experiments to broaden our knowledge on roles of PHLDA1 in functioning of the mitochondria. One of the directions, could be finding the connection between MMP and apoptosis in our model, as both hyper- and de-polarization of mitochondrial membrane were associated with this type of cell death (47). Also, more insights into the two aforementioned cell processes and their relationships with mitochondria can be analyzed in experiments focusing on changes of levels of such proteins as BAX, BAK, BCL-2, BCL-xl, MCL-1, MFN 1/2, OPA1, DRP1 and PINK1 in clones with downregulation of PHLDA1.

In conclusion, our findings show that in IMR-32 cells, the PHLDA1 protein affects mRNA and protein levels of AURKA and NTRK2, Aurora A- and Akt-activating phosphorylations, ATP levels and mitochondrial membrane potential. Our data showing that the protein may be pro-apoptotic and limiting autophagosome formation add to an ongoing debate on roles of PHLDA1 in tumor cells.

\section{Acknowledgements}

We thank Dr R. Reisfeld for providing us with the hybridoma cell line producing the 14G2a mAb. We thank Dr L. Raffaghello for the HTLA230 cell line. We are grateful to Dr M. Bzowska (Department of Immunology of the Faculty of Biochemistry, 
Biophysics and Biotechnology, Jagiellonian University) for help in flow cytometry analyses. We acknowledge that Iwona Nowak, M.Sc., from the Laboratory of Molecular Genetics and Virology (Faculty of Biochemistry, Biophysics and Biotechnology, Jagiellonian University) searched literature to find sequences of starters NTRK1, BDNF, NGF, DKK 3 and designed starters $I D 2$ to detect expression of by RT-qPCR. We are grateful for that. This study was supported by the grant no. NCN-2012/07/B/NZ1/02808 from the Polish National Science Center (to Hanna Rokita), the grant no. 16/2015 from Research Project Competition for Young Researchers and PhD Students of the Faculty of Biochemistry, Biophysics and Biotechnology Jagiellonian University (to Małgorzata Durbas), and DS/8/WBBiB UJ. Faculty of Biochemistry, Biophysics and Biotechnology is a partner of the Leading National Research Center (KNOW) supported by the Ministry of Science and Higher Education.

\section{References}

1. Park CG, Lee SY, Kandala G, Lee SY and Choi Y: A novel gene product that couples TCR signaling to Fas(CD95) expression in activation-induced cell death. Immunity 4: 583-591, 1996.

2. Kuske MD and Johnson JP: Assignment of the human PHLDA1 gene to chromosome $12 \mathrm{q} 15$ by radiation hybrid mapping. Cytogenet Cell Genet 89: 1, 2000.

3. Neef R, Kuske MA, Pröls E and Johnson JP: Identification of the human PHLDA1/TDAG51 gene: Down-regulation in metastatic melanoma contributes to apoptosis resistance and growth deregulation. Cancer Res 62: 5920-5929, 2002.

4. Hayashida N, Inouye S, Fujimoto M, Tanaka Y, Izu H, Takaki E, Ichikawa H, Rho J and Nakai A: A novel HSF1-mediated death pathway that is suppressed by heat shock proteins. EMBO J 25 4773-4783, 2006.

5. Nagai MA, Fregnani JH, Netto MM, Brentani MM and Soares FA: Down-regulation of PHLDA1 gene expression is associated with breast cancer progression. Breast Cancer Res Treat 106: 49-56, 2007.

6. Coutinho-Camillo CM, Lourenço SV, Nonogaki S, Vartanian JG, Nagai MA, Kowalski LP and Soares FA: Expression of PAR-4 and PHLDA1 is prognostic for overall and disease-free survival in oral squamous cell carcinomas. Virchows Arch 463: 31-39, 2013.

7. Murata T, Sato T, Kamoda T, Moriyama H, Kumazawa Y and Hanada N: Differential susceptibility to hydrogen sulfide-induced apoptosis between PHLDA1-overexpressing oral cancer cell lines and oral keratinocytes: Role of PHLDA1 as an apoptosis suppressor. Exp Cell Res 320: 247-257, 2014

8. Park ES, Kim J, Ha TU, Choi JS, Soo Hong K and Rho J: TDAG51 deficiency promotes oxidative stress-induced apoptosis through the generation of reactive oxygen species in mouse embryonic fibroblasts. Exp Mol Med 45: e35, 2013.

9. Hinz T, Flindt S, Marx A, Janssen O and Kabelitz D: Inhibition of protein synthesis by the $\mathrm{T}$ cell receptor-inducible human TDAG51 gene product. Cell Signal 13: 345-352, 2001.

10. Johnson EO, Chang KH, de Pablo Y, Ghosh S, Mehta R, Badve S and Shah K: PHLDA1 is a crucial negative regulator and effector of Aurora A kinase in breast cancer. J Cell Sci 124: 2711-2722, 2011.

11. Otto T, Horn S, Brockmann M, Eilers U, Schüttrumpf L, Popov N, Kenney AM, Schulte JH, Beijersbergen R, Christiansen H, et al: Stabilization of N-Myc is a critical function of Aurora A in human neuroblastoma. Cancer Cell 15: 67-78, 2009.

12. Shang X, Burlingame SM, Okcu MF, Ge N, Russell HV, Egler RA, David RD, Vasudevan SA, Yang J and Nuchtern JG: Aurora $\mathrm{A}$ is a negative prognostic factor and a new therapeutic target in human neuroblastoma. Mol Cancer Ther 8: 2461-2469, 2009.

13. Brockmann M, Poon E, Berry T, Carstensen A, Deubzer HE, Rycak L, Jamin Y, Thway K, Robinson SP, Roels F, et al: Small molecule inhibitors of aurora-a induce proteasomal degradation of N-myc in childhood neuroblastoma. Cancer Cell 24: 75-89, 2013.
14. Mossé YP, Lipsitz E, Fox E, Teachey DT, Maris JM, Weigel B, Adamson PC, Ingle MA, Ahern $\mathrm{CH}$ and Blaney SM: Pediatric phase I trial and pharmacokinetic study of MLN8237, an investigational oral selective small-molecule inhibitor of Aurora kinase A: A Children's Oncology Group Phase I Consortium study. Clin Cancer Res 18: 6058-6064, 2012.

15. Huang $M$ and Weiss WA: Neuroblastoma and MYCN. Cold Spring Harb Perspect Med 3: a014415, 2013.

16. Dhillon S: Dinutuximab: First global approval. Drugs 75: 923-927, 2015.

17. Horwacik I, Durbas M, Boratyn E, Węgrzyn P and Rokita H: Targeting GD2 ganglioside and Aurora A kinase as a dual strategy leading to cell death in cultures of human neuroblastoma cells. Cancer Lett 341: 248-264, 2013.

18. Kowalczyk A, Gil M, Horwacik I, Odrowaz Z, Kozbor D and Rokita H: The GD2-specific 14G2a monoclonal antibody induces apoptosis and enhances cytotoxicity of chemotherapeutic drugs in IMR-32 human neuroblastoma cells. Cancer Lett 281: 171-182, 2009.

19. Wang Y, Sun H, Wang Z, Liu M, Qi Z, Meng J, Sun J and Yang G: Aurora-A: A potential DNA repair modulator. Tumour Biol 35: 2831-2836, 2014

20. Dorn GW II and Kitsis RN: The mitochondrial dynamismmitophagy-cell death interactome: Multiple roles performed by members of a mitochondrial molecular ensemble. Circ Res 116: 167-182, 2015.

21. Riccardi C and Nicoletti I: Analysis of apoptosis by propidium iodide staining and flow cytometry. Nat Protoc 1: 1458-1461, 2006.

22. Stock C, Bozsaky E, Watzinger F, Poetschger U, Orel L, Lion T, Kowalska A and Ambros PF: Genes proximal and distal to MYCN are highly expressed in human neuroblastoma as visualized by comparative expressed sequence hybridization. Am J Pathol 172: 203-214, 2008. Erratum in: Am J Pathol 172: 1153 , 2008.

23. Wang L, Wang G, Yang D, Guo X, Xu Y, Feng B and Kang J: Euphol arrests breast cancer cells at the G1 phase through the modulation of cyclin D1, p21 and p27 expression. Mol Med Rep 8: 1279-1285, 2013.

24. Durbas M, Horwacik I, Boratyn E, Kamycka E and Rokita H: GD2 ganglioside specific antibody treatment downregulates $\mathrm{PI} 3 \mathrm{~K} / \mathrm{Akt} / \mathrm{mTOR}$ signaling network in human neuroblastoma cell lines. Int J Oncol 47: 1143-1159, 2015.

25. Horwacik I, Durbas M, Boratyn E, Sawicka A, Węgrzyn P, Krzanik S, Górka A, Drożniak J, Augustyniak E, Kowalczyk A, et al: Analysis of genes involved in response to doxorubicin and a GD2 ganglioside-specific 14G2a monoclonal antibody in IMR-32 human neuroblastoma cells. Acta Biochim Pol 62: 423-433, 2015.

26. Matsumura S, Terao M, Murota $\mathrm{H}$ and Katayama I: Th2 cytokines enhance TrkA expression, upregulate proliferation, and downregulate differentiation of keratinocytes. J Dermatol Sci 78: 215-223, 2015.

27. Bao W, Qiu H, Yang T, Luo X, Zhang H and Wan X: Upregulation of TrkB promotes epithelial-mesenchymal transition and anoikis resistance in endometrial carcinoma. PLoS One 8: e70616, 2013.

28. Zuccato C, Marullo M, Vitali B, Tarditi A, Mariotti C, Valenza M, Lahiri N, Wild EJ, Sassone J, Ciammola A, et al: Brain-derived neurotrophic factor in patients with Huntington's disease. PLoS One 6: e22966, 2011.

29. Gu YM, Ma YH, Zhao WG and Chen J: Dickkopf3 overexpression inhibits pancreatic cancer cell growth in vitro. World J Gastroenterol 17: 3810-3817, 2011.

30. Skalniak A, Boratyn E, Tyrkalska SD, Horwacik I, Durbas M, Lastowska M, Jura J and Rokita H: Expression of the monocyte chemotactic protein-1-induced protein 1 decreases human neuroblastoma cell survival. Oncol Rep 31: 2385-2392, 2014.

31. Dupasquier S, Delmarcelle AS, Marbaix E, Cosyns JP, Courtoy PJ and Pierreux CE: Validation of housekeeping gene and impact on normalized gene expression in clear cell renal cell carcinoma: Critical reassessment of YBX3/ZONAB/CSDA expression. BMC Mol Biol 15: 9, 2014.

32. Smith PK, Krohn RI, Hermanson GT, Mallia AK, Gartner FH, Provenzano MD, Fujimoto EK, Goeke NM, Olson BJ and Klenk DC: Measurement of protein using bicinchoninic acid. Anal Biochem 150: 76-85, 1985.

33. Edsjö A, Nilsson H, Vandesompele J, Karlsson J, Pattyn F, Culp LA, Speleman F and Påhlman S: Neuroblastoma cells with overexpressed MYCN retain their capacity to undergo neuronal differentiation. Lab Invest 84: 406-417, 2004. 
34. Maris JM: Unholy matrimony: Aurora A and N-Myc as malignant partners in neuroblastoma. Cancer Cell 15: 5-6, 2009.

35. Yang H, He L, Kruk P, Nicosia SV and Cheng JQ: Aurora-A induces cell survival and chemoresistance by activation of Akt through a p53-dependent manner in ovarian cancer cells. Int J Cancer 119: 2304-2312, 2006.

36. Brodeur GM, Minturn JE, Ho R, Simpson AM, Iyer R, Varela CR, Light JE, Kolla V and Evans AE: Trk receptor expression and inhibition in neuroblastomas. Clin Cancer Res 15: 3244-3250, 2009.

37. Tacconelli A, Farina AR, Cappabianca L, Desantis G, Tessitore A, Vetuschi A, Sferra R, Rucci N, Argenti B, Screpanti I, et al: TrkA alternative splicing: A regulated tumor-promoting switch in human neuroblastoma. Cancer Cell 6: 347-360, 2004.

38. Haapasalo A, Sipola I, Larsson K, Akerman KE, Stoilov P, Stamm S, Wong G and Castren E: Regulation of TRKB surface expression by brain-derived neurotrophic factor and truncated TRKB isoforms. J Biol Chem 277: 43160-43167, 2002.

39. Li Z, Tan F, Liewehr DJ, Steinberg SM and Thiele CJ: In vitro and in vivo inhibition of neuroblastoma tumor cell growth by AKT inhibitor perifosine. J Natl Cancer Inst 102: 758-770, 2010.

40. Li Z, Jaboin J, Dennis PA and Thiele CJ: Genetic and pharmacologic identification of Akt as a mediator of brain-derived neurotrophic factor/TrkB rescue of neuroblastoma cells from chemotherapy-induced cell death. Cancer Res 65: 2070-2075, 2005 .
41. Motti ML, De Marco C, Califano D, Fusco A and Viglietto G: Akt-dependent T198 phosphorylation of cyclin-dependent kinase inhibitor p27kip1 in breast cancer. Cell Cycle 3: 10741080, 2004.

42. Viglietto G, Motti ML and Fusco A: Understanding p27(kip1) deregulation in cancer: Down-regulation or mislocalization. Cell Cycle 1: 394-400, 2002.

43. Perry SW, Norman JP, Barbieri J, Brown EB and Gelbard HA: Mitochondrial membrane potential probes and the proton gradient: A practical usage guide. Biotechniques 50: 98-115, 2011.

44. Mizushima N, Yoshimori T and Levine B: Methods in mammalian autophagy research. Cell 140: 313-326, 2010.

45. Li MX and Dewson G: Mitochondria and apoptosis: Emerging concepts. F1000Prime Rep 7: 42, 2015.

46. Zhao J, Zhang J, Yu M, Xie Y, Huang Y, Wolff DW, Abel PW and Tu Y: Mitochondrial dynamics regulates migration and invasion of breast cancer cells. Oncogene 32: 4814-4824, 2013.

47. Gottlieb E, Vander Heiden MG and Thompson CB: Bcl-x(L) prevents the initial decrease in mitochondrial membrane potential and subsequent reactive oxygen species production during tumor necrosis factor alpha-induced apoptosis. Mol Cell Biol 20: 5680-5689, 2000 